\title{
The Anderson impurity model with a narrow-band host: from orbital physics to the Kondo effect
}

\author{
Steffen Schäfer ${ }^{1}$ \\ ${ }^{1}$ Aix-Marseille Université and Institut Matériaux Microélectronique Nanosciences de Provence (IM2NP), \\ Faculté des Sciences de St Jérôme, $1339^{7}$ Marseille, France
}

(Dated: September 27, 2010; revised manuscript March 25, 2011)

\begin{abstract}
A particle-hole symmetric Anderson impurity model with a metallic host of narrow bandwidth is studied within the framework of the local moment approach. The resultant single-particle spectra are compared to unrestricted Hartree-Fock, second order perturbation theory about the noninteracting limit, and Lanczos spectra by Hofstetter and Kehrein. Rather accurate analytical results explain the spectral evolution over almost the entire range of interactions. These encompass, in particular, a rationale for the four-peak structure observed in the low-energy sector of the Lanczos spectra in the moderate-coupling regime. In weak coupling, the spectral evolution is governed by orbital effects, while in the strong coupling Kondo limit, the model is shown to connect smoothly to the generic Anderson impurity with a flat and infinitely wide hybridization band.
\end{abstract}

PACS numbers: 71.27.+a Strongly correlated electron systems; heavy fermions $-71.28 .+\mathrm{d}$ Narrow-band systems; intermediate-valence solids - 71.55.-i Impurity and defect levels - 75.20.Hr Local moment in compounds and alloys; Kondo effect, valence fluctuations, heavy fermions

\section{INTRODUCTION}

Five decades of intense experimental and theoretical research have boosted the Anderson impurity model $(\mathrm{AIM})^{11}$ far beyond the scope of the "localized magnetic states in metals" that it was initially designed for. In the first three decades, its concept of a single level with on-site Coulomb repulsion coupled to a host without electronic interactions was mainly used to describe magnetic transition metal impurities dissolved in otherwise nonmagnetic bulk metals. The last two decades' extraordinary progress in nanotechnology, however, brought a myriad of new and rather surprising implementations of what in the meantime had become one of the theorists' favourite toys, ranging from tunable quantum $\operatorname{dot}^{2 \sqrt{213}}$ over carbon nanotubes $\frac{415}{4}$ and adsorbed organic molecules ${ }^{6}$ to single-electron ${ }^{7}$ or single-molecule transistors $\left[{ }^{8}\right.$

The vast majority of theoretical work, comprehensively reviewed in Ref. 9, focuses on AIMs with metallic hosts of large bandwidth. In the limit of infinite bandwidth, the exact static and thermodynamic properties can be deduced from the Bethe ansatz solution $[10,12$ A powerful and versatile alternative is provided by the numerical renormalization group (NRG) 13114 recent extensions of which have also been able to address the dynamics of the model to excellent accuracy 15

Yet another field of application is the Mott metal-toinsulator transition in high spatial dimensions: ${ }^{2224}$ here, dynamical mean-field theory (DMFT) ${ }^{23}$ reduces the at first sight unrelated problem of interacting electrons on a high-dimensional lattice to an effective AIM immersed in a bath of identical sites whose properties have to be determined self-consistently. It was within this context that the possibility of an AIM with a metallic host of narrow bandwidth was first evoked ${ }^{25}$ since it naturally arises, in the vicinity of the Mott-Hubbard transition, $\frac{26}{26}$ within the now widely accepted scenario of a metallic state surrounded by a preformed gap ${ }^{23}$ At the time, W. Hofstetter and S. Kehrein argued, on grounds of their Lanczosdetermined single-particle spectra for a corresponding $\mathrm{AIM}, \frac{25}{25}$ that this incipient gap might be populated by localized states. Although these states have not actually been observed inside the preformed gap of the infinitedimensional Hubbard model, recent high-resolution dynamic density-matrix renormalization group (DDMRG) data 27.29 do indeed show very narrow features on the inner band edges of the Hubbard satellites in the appropriate regime of interactions.

Independent from the relevance for the Mott-Hubbard transition, several questions about these sharp features may arise: (i) what are the underlying physical processes? (ii) do these processes depend on correlations within the DMFT bath? (iii) why do these features disappear for both, small and large values of the Coulomb repulsion? (iv) are they related - and if yes, in which manner - to the series of peaks observed in the low-energy sector of Lanczos-determined spectra of an AIM with a correlationless narrow-band host?25 and (v) why, in the latter case, are the peaks organized in a four-set structure?

Some of the above questions have already been addressed in a previous article $\stackrel{30}{,}$ in which the AIM was mainly studied perturbatively about the noninteracting limit; others, in particular those concerning the rich lowenergy structure at moderate interaction strengths or the strong-coupling Kondo regime, lie out of reach for perturbative approaches and remain unanswered thus far. It is these questions, among others, that shall be addressed in the present article, primarily within the framework of the so-called local moment approach (LMA). This nonperturbative many-body Green function formalism, developed by D. Logan and co-workers, ${ }^{19131 / 32}$ introduces the concept of local moments from the outset. At pure 
mean-field level, this would lead to a doubly degenerate ground-state, as appropriate for an insulator, but in manifest contradiction to the Kondo singlet observed for impurities hosted in metals. The LMA aims to transcend this deficiency by accounting for dynamical tunneling processes between the two mean-field ground-states, at a rate which has to be determined in consistency with Fermi-liquid behaviour on the lowest energy scale. The resulting formalism is equally well adapted to impurities in metallic ${ }^{31}$ and insulating ${ }^{33}$ hosts, be it in the particle-hole symmetric limit ${ }^{31}$ or away from it ${ }^{32}$ The LMA has so far been applied and adapted to a variety of impurity and lattice problems with strong electronic correlations, where its ability to cope with essentially all interaction regimes and to correctly describe all energy scales has proven very valuable. $[4 \sqrt[39]{3 n}$ the present case of an impurity hosted in a narrow band, the LMA has to be generalized by using a renormalized, sum-rule compliant version of the original ladder-sum propagator for the transverse spin fluctuations. This extension is necessary, especially in the regime of moderate interaction strengths, to correctly capture the subtle low-energy dynamics (see Secs. III A and IV B).

The article is outlined as follows: after a brief presentation of the model, Sec. II defines the narrow-band regime and presents two different mean-field solutions. Sec. III starts with a review of the LMA, followed by a discussion of the physically relevant transverse spin fluctuations and of two important sum rules for the associated polarization propagator; its last paragraph is dedicated to the self-energy approximation implemented in practice. Sec. IV presents the LMA impurity spectra for different regimes defined by the strength of the on-site Coulomb interaction $U$; the spectra are compared to corresponding Hartree-Fock or perturbation theory results and, where available, to Hofstetter and Kehrein's Lanczosdetermined spectra. ${ }^{25}$ A Conclusion section closes the paper.

\section{HAMILTONIAN AND MEAN FIELD THEORIES}

The Hamiltonian for the AIM is given in standard notation by

$$
\begin{aligned}
\hat{H}= & \sum_{\mathbf{k} \sigma} \varepsilon_{\mathbf{k}} \hat{n}_{\mathbf{k} \sigma}+\sum_{\sigma} \varepsilon_{i} \hat{n}_{i \sigma}+U \hat{n}_{i \uparrow} \hat{n}_{i \downarrow} \\
& +\sum_{\mathbf{k} \sigma}\left(V_{i \mathbf{k}} c_{i \sigma}^{+} c_{\mathbf{k} \sigma}+\text { h.c. }\right)
\end{aligned}
$$

where the first term describes electrons (of spin $\sigma=\uparrow, \downarrow$ ) in a metallic host band of dispersion $\varepsilon_{\mathbf{k}}$. The following two terms refer to the impurity, with $\varepsilon_{i}$ the impurity level and $U$ the on-site Coulomb interaction. The final term describes the one-electron hybridization between the impurity and host.

Throughout this article, as in Refs. 25 and 30 , the particle-hole symmetric AIM, obtained by setting $\varepsilon_{i}=$
$-U / 2$, will be studied. In this case, the empty and doubly occupied impurity states are degenerate, whence for all interaction strengths the Fermi level remains fixed at its noninteracting value and the impurity charge is $n_{i}=\left\langle\hat{n}_{i \uparrow}+\hat{n}_{i \downarrow}\right\rangle=1$. Regardless of the interaction strength, single-particle spectra are thus symmetric with respect to the Fermi level, $\omega=0$. Persistent charge fluctuations guarantee the system's metallic character, allowing for the possibility to recast the exact single-particle impurity Green function as an infinite-order perturbation series, with each diagram depending solely on $U$ and the noninteracting Green function

$$
g(\omega)=\left[\omega+\mathrm{i} 0^{+} \operatorname{sgn}(\omega)-\Delta(\omega)\right]^{-1} .
$$

In the latter expression, $\Delta(\omega)=\Delta_{R}(\omega)-i \operatorname{sgn}(\omega) \Delta_{I}(\omega)$ stands for the hybridization function

$$
\Delta(\omega)=\sum_{\mathbf{k}} \frac{\left|V_{i \mathbf{k}}\right|^{2}}{\omega+\mathrm{i} 0^{+} \operatorname{sgn}(\omega)-\varepsilon_{\mathbf{k}}}
$$

which condenses all relevant information about the host dispersion $\varepsilon_{\mathbf{k}}$ and the hybridization matrix elements $V_{i \mathbf{k}}$.

In this section, the single-particle impurity spectra of the AIM will be calculated in two different meanfield descriptions: Restricted Hartree-Fock (RHF), on the one hand, implements spin symmetry from the outset via identical impurity occupation numbers for both spin species; unrestricted Hartree Fock (UHF), on the other hand, seeks to determine the occupation numbers selfconsistently - thus allowing for solutions with different impurity occupancies for $\uparrow$ and $\downarrow$-spins at an intermediate stage - and restores the full spin symmetry only at the very end.

In both versions of the theory, the (causal) singleparticle impurity Green function $\mathcal{G}_{i \sigma}(\omega)=\operatorname{Re} \mathcal{G}_{i \sigma}(\omega)-$ $i \operatorname{sgn}(\omega) \pi D_{i \sigma}^{0}(\omega)$ can be deduced via standard techniques, e.g., the equation-of-motion method, after Hartree-Fock factorizing the 2-body term in the Hamiltonian (1), $\hat{n}_{i \uparrow} \hat{n}_{i \downarrow} \simeq \hat{n}_{i \uparrow}\left\langle\hat{n}_{i \downarrow}\right\rangle+\left\langle\hat{n}_{i \uparrow}\right\rangle \hat{n}_{i \downarrow}-\left\langle\hat{n}_{i \uparrow}\right\rangle\left\langle\hat{n}_{i \downarrow}\right\rangle$, yielding

$$
\mathcal{G}_{i \sigma}(\omega)=\left[\omega+\mathrm{i} 0^{+} \operatorname{sgn}(\omega)-\varepsilon_{i \sigma}-\Delta(\omega)\right]^{-1}
$$

where $\varepsilon_{i \sigma}=\varepsilon_{i}+\frac{U}{2}\left(n_{i}-\sigma \mu\right)$ denotes the Hartree-Fock corrected impurity level. In the particle-hole symmetric AIM, where $\varepsilon_{i}=-U / 2$ and $n_{i}=\left\langle\hat{n}_{i \uparrow}+\hat{n}_{i \downarrow}\right\rangle=1$, the Hartree-Fock corrected impurity level solely depends on the impurity moment $\mu=\left\langle\hat{n}_{i \uparrow}-\hat{n}_{i \downarrow}\right\rangle$, viz.

$$
\varepsilon_{i \sigma}=-\frac{U}{2} \sigma \mu
$$

with $\sigma=+(-)$ for $\uparrow(\downarrow)$ spins. As a consequence of the Hamiltonian's invariance under spin inversion, solutions with a nonvanishing magnetic moment are doubly degenerate, $\mu=+|\mu|$ and $-|\mu|$.

The present paper focuses on AIMs with narrow metallic host bands, whose width is much smaller than the hybridization strength at the Fermi level, $\Delta_{0}=\Delta_{\mathrm{I}}(\omega=0)$. 
The physics of such a narrow-band AIM is naturally rather insensitive to the precise form of the hybridization, meaning that, without loss of generality, $\Delta_{\mathrm{I}}(\omega)$ may be assumed to consist of a single flat band of intensity $\Delta_{0}$, ranging from $-D$ to $+D$, with $D \ll \Delta_{0}$. As for any timeordered Green function the real part follows via Hilbert transform,

$$
\Delta_{\mathrm{R}}(\omega)=\mathcal{P} \int_{-\infty}^{+\infty} \frac{\mathrm{d} \omega^{\prime}}{\pi} \frac{\Delta_{\mathrm{I}}\left(\omega^{\prime}\right)}{\omega-\omega^{\prime}},
$$

so that in total

$$
\Delta(\omega)=\frac{\Delta_{0}}{\pi} \ln \left|\frac{\omega+D}{\omega-D}\right|-\mathrm{i} \Delta_{0} \operatorname{sgn}(\omega) \theta(D-|\omega|) .
$$

\section{A. Restricted Hartree-Fock (RHF)}

The spin-reversal invariance of the Anderson Hamiltonian (1) implies that the average number of $\uparrow$ and $\downarrow$-spin electrons on the impurity has to be the same for any interaction strength. RHF theory acknowledges this fact from the outset by enforcing $\left\langle\hat{n}_{i \uparrow}\right\rangle=\left\langle\hat{n}_{i \downarrow}\right\rangle$ for all interactions strengths, thus entailing $\varepsilon_{i \sigma} \equiv 0$ in eq. (4). As a result, RHF recovers the noninteracting Green function for both spin species and all interaction strengths:

$$
G_{\sigma}^{\mathrm{RHF}}(\omega) \equiv g(\omega)=\left[\omega+\mathrm{i} 0^{+} \operatorname{sgn}(\omega)-\Delta(\omega)\right]^{-1}
$$

The associated single-particle spectrum $D_{i \sigma}^{0}(\omega)$ consists of two contributions: (i) a continuum for $\omega \in$ $[-D, D]$, arising from the nonzero imaginary part of the hybridization; and (ii) two poles, one lying above and the other symmetrically below the continuum. The fraction of spectral weight residing in the poles depends strongly on the bandwidth of the host metal. In the usual wideband model, $D \gg \Delta_{0}$, most of the spectral intensity is concentrated in the single-particle band, while the poles are exponentially weak and hence irrelevant in practice.

The situation is, however, radically different in the present narrow-band model, defined by $D \ll \Delta_{0}$. Here, almost the entire spectral intensity resides in the poles, occurring at frequencies $\pm \omega_{0}$ far outside the band. By analogy with an $\mathrm{H}_{2}$ molecule, these poles can be viewed as a bonding and an anti-bonding orbital, thus suggesting that the narrow host band to which the impurity is coupled behaves effectively as a single site or level. $\stackrel{25 / 40}{ }$

In this case, the pole frequencies and weights can be obtained to good accuracy from eq. (8) by using the expansion $\Delta_{\mathrm{R}}(\omega) \sim(2 / \pi) \Delta_{0} D / \omega$, valid for $|\omega| \gg D$ :

$$
\begin{aligned}
\omega_{0} & \simeq \sqrt{\frac{2}{\pi} \Delta_{0} D} \\
q & \simeq \frac{1}{2}-\frac{8 D}{\pi \Delta_{0}} .
\end{aligned}
$$

The "bonding energy" $\omega_{0}$ corresponds to the integrated hybridization, or the total hopping between host and impurity $[30]$ subject to $D \ll \omega_{0} \ll \Delta_{0}$, it defines a second low-energy scale relevant in the narrow-band regime.

In the following, it will sometimes be helpful to consider $D$ and $\omega_{0}$ as independent parameters. In particular, in the limit of an infinitely narrow host band, $D \rightarrow 0$, this allows us to treat the host as a single level which couples via a finite bonding energy $\omega_{0}$ to the impurity - a picture henceforth referred to as the two-site approximation.

In addition to the orbital levels of the two-site approximation, the full noninteracting single-particle spectrum encompasses the aforementioned Fermi liquid continuum stemming from the hybridization band on the lowest energy scale, $|\omega| \leq D$. Its integrated weight is of the order $\mathcal{O}\left(D / \Delta_{0}\right)$ and thus weak in the narrow-band regime.

The RHF description, naturally exact in the noninteracting limit, is expected to break down if $U$ is much larger than the "molecular" bonding energy $\omega_{0}$ : in this case, the extra electron probed by $G_{\sigma}$ is most likely to be introduced on an already singly occupied impurity which involves an energy cost of the order of the interaction strength $U$; the single-particle spectra will then be dominated by Hubbard poles separated by the Coulomb interaction $U$ rather than the molecular orbitals at $\omega= \pm \omega_{0}$.

\section{B. Unrestricted Hartree-Fock (UHF)}

In UHF theory, the magnetic moment residing on the impurity will be determined self-consistently from the impurity Green function $\mathcal{G}_{i \sigma}$ itself. For small interaction strengths, it is found to be zero, and UHF recovers the noninteracting solution $g$. This nonmagnetic solution becomes unstable above some critical interaction $U_{0}^{\mathrm{c}}-$ which turns out to be related to the "molecular" bonding energy $\omega_{0}$ - and UHF then converges to a solution with a finite impurity moment. The Hamiltonian (1) is, however, still invariant under spin inversions, thus guaranteeing for any mean-field ground state with positive moment $\mu=+\mu_{0}$ the existence of another degenerate ground state with opposite moment, $\mu=-\mu_{0}$. Subsequently, quantum mechanical tunneling processes between these mean-field ground states ensure their occurrence with equal probability:

$$
G^{\mathrm{UHF}}(\omega)=\frac{1}{2}\left[\mathcal{G}_{\mathrm{A} \sigma}(\omega)+\mathcal{G}_{\mathrm{B} \sigma}(\omega)\right]
$$

Throughout the present paper and without loss of generality, A- (B-) type impurities are assumed to be predominantly $\uparrow(\downarrow)$ spin occupied, implying $\mu_{0} \geq 0$. The $\uparrow \downarrow$ spin symmetry of the Hamiltonian entails $\mathcal{G}_{\mathrm{A} \sigma}(\omega)=\mathcal{G}_{\mathrm{B}-\sigma}(\omega)$, thus guaranteeing the spin independence of $G^{\mathrm{UHF}}(\omega)$ in eq. 10 .

The necessity of the mixing process 10 becomes particularly obvious in the atomic limit, defined by vanishing hybridization matrix elements, $V_{i \mathbf{k}} \equiv 0$. In this limit, the impurity propagator is $G^{\mathrm{AL}}(\omega)=\frac{1}{2}\left(\left[z+\frac{U}{2}\right]^{-1}+\left[z-\frac{U}{2}\right]^{-1}\right)$ [with $\left.z=\omega+\mathrm{i} 0^{+} \operatorname{sgn}(\omega)\right]$. Each of the two contributions in eq. 10 yields one term of this exact result [via 
$\Delta(\omega) \equiv 0$ and $\left.\mu_{0}=1\right]$ : an $\uparrow$-spin electron can only be retrieved from an A-type impurity (first term), and can only be added to a B-type impurity (second term).

In practical terms, the Green function $\mathcal{G}_{\mathrm{A} \sigma}(\omega)=$ $\operatorname{Re} \mathcal{G}_{\mathrm{A} \sigma}(\omega)-i \operatorname{sgn}(\omega) \pi D_{\mathrm{A} \sigma}^{0}(\omega)$ is obtained from eqs. (4) and (5) with an impurity moment $\mu=+\mu_{0}$, calculated self-consistently from

$$
\mu_{0}=\int_{-\infty}^{0} \mathrm{~d} \omega\left[D_{\mathrm{A} \uparrow}^{0}(\omega)-D_{\mathrm{A} \downarrow}^{0}(\omega)\right] ;
$$

$\left[\mathcal{G}_{\mathrm{B} \sigma}(\omega)\right.$ follows equivalently for $\left.\mu=-\mu_{0}\right]$. In Fig. 1 , the

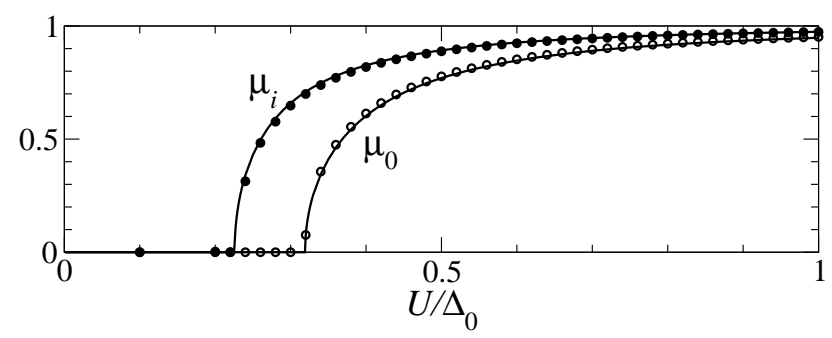

FIG. 1: Magnetic impurity moment vs. Coulomb repulsion $U$ for an AIM with bandwidth $D=0.01 \Delta_{0}$, where $\omega_{0} \simeq 0.08 \Delta_{0}$. Filled circles: numerically determined LMA moment $\mu$, as required by eq. 35 ; open circles: numerically determined UHF moment $\mu_{0}$. Solid lines: corresponding approximate analytical expressions, eqs. (36) and (13). The critical interactions are $U^{\mathrm{c}} \simeq 0.23 \Delta_{0}$ for the LMA, and $U_{0}^{\mathrm{c}} \simeq 0.32 \Delta_{0}$ in UHF.

self-consistent UHF moment $\mu_{0}$, eq. (11), is plotted as a function of the interaction strength $U$ for a narrow-band AIM with host bandwidth $D=0.01 \Delta_{0}$.

The spectral density $D_{\mathrm{A} \downarrow}^{0}(\omega)$ consists of a low-energy continuum for $|\omega|<D$, of net weight $\mathcal{O}\left(D / \Delta_{0}\right)$, arising from the finite imaginary part of the hybridization, and two pole contributions, one above the single-particle band, at $\omega=\omega_{>}>+D$, and the other one below it, at $\omega=-\omega_{<}<-D$. Assuming both poles to occur far outside the single-particle band, the pole frequencies and weights can be obtained rather accurately by expansion of the hybridization function (7), $\Delta_{\mathrm{R}}(\omega) \sim(2 / \pi) \Delta_{0} D / \omega$ :

$$
\begin{aligned}
& \omega_{\gtrless} \simeq \omega_{0}\left(\sqrt{y_{0}^{2}+1} \pm y_{0}\right) \\
& q_{\gtrless} \simeq \frac{\sqrt{y_{0}^{2}+1} \pm y_{0}}{2 \sqrt{y_{0}^{2}+1}}
\end{aligned}
$$

with $y_{0}=U \mu_{0} / 4 \omega_{0}$.

For the above expansion to hold, $\omega_{\gtrless} \gg D$ is required, thus limiting the validity of eq. 12 for the low-energy pole at $\omega=-\omega_{<}$to interactions $U \mu_{0} \ll \frac{4}{\pi} \Delta_{0}$ (while no restrictions follow for the high-energy pole $\omega_{>}$). In this range of interactions, the renormalization effects in the single-particle continuum are still small and the UHF moment $\mu_{0}$ can be obtained very accurately by only retaining the pole contributions of $D_{\mathrm{A} \sigma}^{0}(\omega)$ in the self-consistency equation (11). This yields the following expression which corresponds to the lower solid line in Fig. 1 .

$$
\mu_{0} \simeq \begin{cases}\sqrt{1-\left(U_{0}^{\mathrm{c}} / U\right)^{2}} & \text { for } U>U_{0}^{\mathrm{c}}:=4 \omega_{0} \\ 0 & \text { for } U<U_{0}^{\mathrm{c}}\end{cases}
$$

For $U<U_{0}^{\mathrm{c}}$, the self-consistently determined moment vanishes and the UHF Green function coincides with the noninteracting or RHF solution, eq. (8). Above $U_{0}^{\mathrm{c}}$, by contrast, a finite local moment forms on the impurity, and saturates rapidly as $U$ is increased. With increasing $\mu_{0}$, the pole at $\omega=\omega_{>}$shifts rapidly away from its noninteracting value $\omega_{0}$ towards higher frequencies and gains in intensity. For $U \gg U_{0}^{\mathrm{c}}$, it becomes the upper Hubbard satellite at $\omega_{>} \simeq U / 2$, which overwhelmingly dominates the spectrum $D_{\mathrm{A} \downarrow}^{0}(\omega)$ with a pole weight of $q_{>} \simeq 1-\mathcal{O}\left(\left[\omega_{0} / U\right]^{2}\right)$. Simultaneously, the pole at $\omega=-\omega_{<}$moves from $-\omega_{0}$ towards the lower band edge $-D$ and loses weight. For $U_{0}^{\mathrm{c}} \ll U \lesssim \Delta_{0}$, its position and weight are given to good accuracy by

$$
\begin{aligned}
& \omega_{<} \simeq \frac{2 \omega_{0}^{2}}{U}=: \frac{J}{2}, \\
& q_{<} \simeq\left(\frac{2 \omega_{0}}{U}\right)^{2} .
\end{aligned}
$$

The first equation defines a third low-energy scale, $J=$ $4 \omega_{0}^{2} / U$, which lies between bandwidth and bonding energy, $D \ll J \ll \omega_{0}$, and accounts for the antiferromagnetic exchange between impurity and host.

If $U$ is increased to values of the order of $\Delta_{0}$, the antiferromagnetic exchange $J$ approaches the bandwidth $D$, and the above analysis - while still valid for the Hubbard level at $\omega=\omega_{>}$- breaks down for the lowenergy pole at $\omega=-\omega_{<}$. If, like in the present case, the hybridization band $\Delta_{I}(\omega)$ has a discontinuity at the lower band edge $-D$, the logarithmic divergence of the related real part, $\Delta_{R}(\omega)$, still guarantees the existence of a low-energy pole; in the present model with a flat hybridization band it occurs, for $U \gg \Delta_{0}$ (where $\mu_{0} \simeq 1$ ), exponentially close to the band edge and carries exponentially small weight, which renders it insignificant in practice:

$$
\begin{aligned}
& \omega_{<} \simeq D\left[1+2 \exp \left(-\frac{\pi U}{2 \Delta_{0}}\right)\right] \\
& q_{<} \simeq \frac{2 \pi D}{\Delta_{0}} \exp \left(-\frac{\pi U}{2 \Delta_{0}}\right)
\end{aligned}
$$

Finally, according to eq. 100, the full UHF impurity spectrum can be obtained by superposing $D_{\mathrm{A} \downarrow}^{0}(\omega)$ and $D_{\mathrm{B} \downarrow}^{0}(\omega)$, the latter of which follows by symmetry, $D_{\mathrm{B} \downarrow}^{0}(\omega)=D_{\mathrm{A} \uparrow}^{0}(\omega)$. As illustrated in Fig. 2, $D^{\mathrm{UHF}}(\omega)$ consist of a continuum for $|\omega|<D$, and two pairs of poles at $\omega= \pm \omega_{>}$and $\omega= \pm \omega_{<}$. Before the onset 

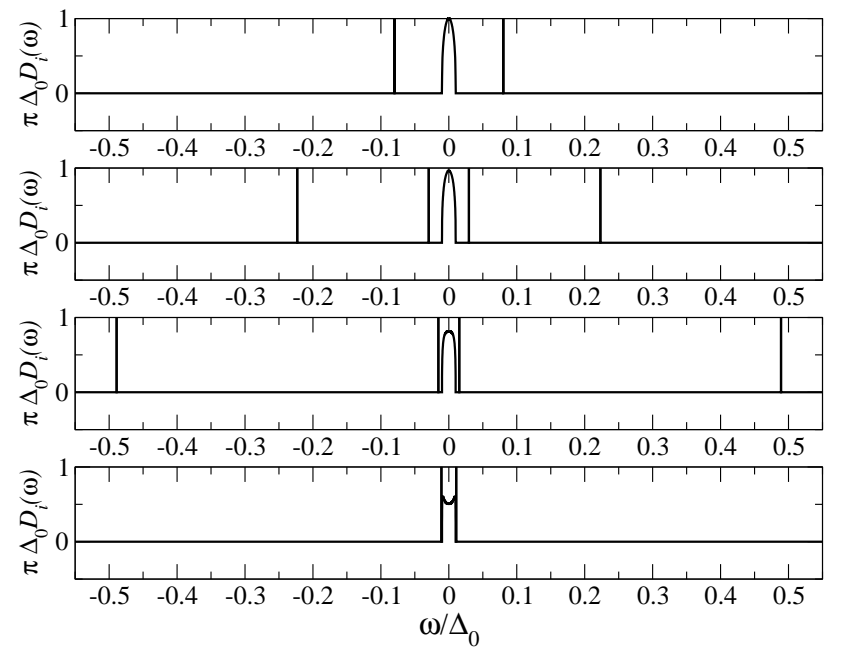

FIG. 2: UHF impurity spectra, $\pi \Delta_{0} D^{\mathrm{UHF}}(\omega)$ vs. $\omega / \Delta_{0}$, for bandwidth $D=0.01 \Delta_{0}$, i.e., $\omega_{0} \simeq 0.08 \Delta_{0}$ and $U_{0}^{\mathrm{c}} \simeq 0.32 \Delta_{0}$, and interaction strengths $U / \Delta_{0}=0,0.5,1,2$ (from top to bottom). In the bottom panel, the Hubbard satellites at $\omega \simeq$ $\pm U / 2= \pm \Delta_{0}$ lie off plot range. Discrete levels are represented by vertical lines. The Fermi energy is $\omega=0$.

of moment formation, i.e., for $U<U_{0}^{\mathrm{c}}$, the two pairs merge into the single pair of molecular orbitals shown in the first graph, at $\omega= \pm \omega_{0}$ and of net weight $q \sim 1 / 2$ each, and UHF coincides with the noninteracting solution. As $U$ exceeds $U_{0}^{\mathrm{c}}$, the orbitals split up progressively into a stronger growing high- and a weaker growing lowenergy component, as depicted in the second and third graphs of Fig. 2. In the second panel, where $U=0.5 \Delta_{0}$ is only moderately greater than $U_{0}^{\mathrm{c}} \simeq 0.32 \Delta_{0}$, the impurity moment is already well established, $\mu_{0} \simeq 0.78$, and the "Hubbard satellites" at $\omega \simeq \pm 0.22 \Delta_{0}$ are not far from their terminal position, $\omega \simeq \pm U / 2= \pm 0.25 \Delta_{0}$, carrying together more than $88 \%$ of the spectral intensity. Approximately a further $11 \%$ of the weight reside in the low-frequency poles at $\omega \simeq \pm 0.030 \Delta_{0}$ (expected at $\omega \simeq \pm J / 2 \simeq \pm 0.025 \Delta_{0}$ from eq. (14a)), while the central low-energy continuum carries only less than $1 \%$ of the spectral intensity. In the third graph, where $U=\Delta_{0}$, roughly $98 \%$ of the spectral weight reside in the Hubbard satellites at $\omega \simeq \pm 0.49 \Delta_{0}$, while the low-energy poles at $\omega \simeq \pm 0.015 \Delta_{0}$ appear close to the central continuum, the latter being strongly renormalized from its noninteracting shape. Finally, these renormalization effects are still enhanced for $U=2 \Delta_{0}$ (bottom panel), not yet fully in the strong coupling regime, resulting in a considerable violation of the Friedel sum rule (see below); here, almost all intensity resides in the Hubbard satellites (off plot range), and exponentially weak lowenergy poles, with total weight $0.3 \%$, are located slightly outside the central continuum [see eqs. [15]].

The above scenario, with a spectral evolution governed by $\omega_{0} \simeq \sqrt{2 \Delta_{0} D / \pi}$ in weak coupling, and by $U$ and $J=4 \omega_{0}^{2} / U$ in moderate coupling, $U_{0}^{\mathrm{c}} \ll U \lesssim \Delta_{0}$, con- curs qualitatively with second order perturbation theory in $U(2 \mathrm{PT}), \frac{30}{30}$ and can be rationalized in terms of a simple two-site model in which the host is caricatured by a single level coupled to the impurity 40 Despite these encouraging results, UHF suffers from several severe limitations: (i) in the two-site limit, $D \rightarrow 0$ albeit with finite $\omega_{0}$, exactly captured by $2 \mathrm{PT}$, the lowenergy single-particle levels are expected at $\omega \simeq \pm 3 J / 2$ for $U \gg U_{0}^{\mathrm{c}}$, i.e., three times the corresponding UHF pole frequency (14a); (ii) the UHF single-particle band, present for $|\omega|<D$, is generally not a Fermi liquid: for interactions above $U_{0}^{\mathrm{c}}$, a nonzero impurity moment $\mu_{0}$ forms, and the UHF zero-frequency behaviour $D^{\mathrm{UHF}}(\omega=$ $0)=\left(1 / \pi \Delta_{0}\right) /\left[1+\left(U \mu_{0} / 2 \Delta_{0}\right)^{2}\right]$ violates the Friedel sum rule ${ }^{9 / 41}$ that compels the impurity spectra of the particlehole symmetric AIM, for arbitrary interaction strengths, to be pinned at the Fermi level to their noninteracting value, $D(\omega=0)=1 / \pi \Delta_{0}$; and (iii), relatedly, due to the absence of dynamics UHF completely fails to capture any of the Kondo physics expected to govern the low-energy spectrum in the strong coupling regime, $U \gg 4 \Delta_{0}$.

The present paper aims to transcend these shortcomings within the framework of the local moment approach (LMA).

\section{LOCAL MOMENT APPROACH (LMA)}

The LMA $A^{1913132}$ expresses the impurity Green function in a formalism employing two self-energies. At pure mean-field level this description reduces to UHF, discussed in the previous section, with each of the selfenergies arising from impurities with predominant $\uparrow$ - or $\downarrow$-spin occupation. For an impurity hosted by an insulator with a sufficiently large gap, this doubly degenerate ground-state is actually observed $33 \mid 42$ Conversely, for a metallic host, dynamical tunneling between the two broken-symmetry states can (and will) still lower the energy and ultimately lead to the formation of a "Kondo" singlet ground-state with fully restored spin symmetry on the longest timescales $\stackrel{3132}{32}$ The tunneling mechanism requires a (virtually) doubly occupied or empty impurity at some intermediate stage, implying that its rate - and the corresponding energy scale - diminish with increasing interaction strength. The LMA incorporates such a mechanism by coupling the single-particle dynamics to energetically low-lying flips of the impurity moment. In order to obtain a successful description of insulating and metallic phases within the same framework, this has to be done in a manner encompassing the possibility of selfconsistent restoration of the spin symmetry at low energies, as necessary for the preservation of Fermi-liquid behaviour on this scale.

The implementation of the LMA follows Refs. 31 and 32. For reasons analogous to those discussed in the context of eq. (10), the full impurity Green function $G$ is again obtained by superposing A- and B-type impurity 
propagators in a spin-rotationally invariant fashion,

$$
G(\omega)=\frac{1}{2}\left[G_{\mathrm{A} \sigma}(\omega)+G_{\mathrm{B} \sigma}(\omega)\right] .
$$

Making use of spin symmetry, $G_{\mathrm{B} \sigma}(\omega)=G_{\mathrm{A}-\sigma}(\omega)$, yields the equivalent expression

$$
G(\omega)=\frac{1}{2}\left[G_{\alpha \uparrow}(\omega)+G_{\alpha \downarrow}(\omega)\right] .
$$

Here, $G$ is independent of the impurity type and the indices $\alpha$ may be suppressed for convenience, meaning that the individual (broken-symmetry) $G_{\sigma}(\omega)$ in eq. $16 \mathrm{~b}$ will henceforth be implicitly considered "A-type", i.e., of impurity moment $\mu \geq 0$, unless stated otherwise. Each of the $G_{\sigma}(\omega)$ can be expressed in terms of a Dyson equation,,$\underline{51}$

$$
G_{\sigma}(\omega)=\left[g^{-1}(\omega)-\tilde{\Sigma}_{\sigma}(\omega)\right]^{-1},
$$

thus defining the two self-energies $\tilde{\Sigma}_{\uparrow}(\omega)$ and $\tilde{\Sigma}_{\downarrow}(\omega)$ central to the present approach. Without loss of generality, the two self-energies can be separated into static and dynamic contributions, $\tilde{\Sigma}_{\sigma}(\omega)=\Sigma_{\sigma}^{\text {st }}+\Sigma_{\sigma}(\omega)$ : diagrammatically, the former are suitably approximated by the Hartree tadpole (while the contribution of the Fock open oyster diagram vanishes), amounting to

$$
\Sigma_{\sigma}^{\mathrm{st}}=-\sigma \frac{U}{2} \int_{-\infty}^{0} \mathrm{~d} \omega\left[D_{\mathrm{A} \uparrow}^{0}(\omega)-D_{\mathrm{A} \downarrow}^{0}(\omega)\right] ;
$$

and the dynamical $\Sigma_{\sigma}(\omega)$ are defined to contain "everything else". Eq. (17) may thus be rephrased as

$$
G_{\sigma}(\omega)=\left[\mathcal{G}_{\sigma}^{-1}(\omega)-\left(\Sigma_{\sigma}^{\mathrm{st}}-\varepsilon_{i \sigma}\right)-\Sigma_{\sigma}(\omega)\right]^{-1} .
$$

This choice is particularly convenient if - as for the LMA - the dynamic self-energy contributions $\Sigma_{\sigma}(\omega)$ are to be diagrammatically constructed from the UHF propagators $\mathcal{G}_{\sigma}$ rather than from the noninteracting $g$, since it entails the sum of the static contributions (in braces) to vanish if the UHF moment $\mu_{0}$, determined self-consistently from eq. (11), is used.

The present two-self-energy description is by now well established and by no means exclusive to the LMA, but likewise emerges in other approaches as, e.g., in the NRG where it occurs for odd iterations of the renormalization group ${ }^{20}$ Nevertheless, it is desirable to connect it to the conventional single self-energy, defined by the Dyson equation $G(\omega)=\left[g^{-1}(\omega)-\Sigma(\omega)\right]^{-1] 3132}$

$$
\begin{aligned}
\Sigma(\omega)= & \frac{1}{2}\left[\tilde{\Sigma}_{\uparrow}(\omega)+\tilde{\Sigma}_{\downarrow}(\omega)\right] \\
& +\frac{\left(\frac{1}{2}\left[\tilde{\Sigma}_{\uparrow}(\omega)-\tilde{\Sigma}_{\downarrow}(\omega)\right]\right)^{2}}{g^{-1}(\omega)-\frac{1}{2}\left[\tilde{\Sigma}_{\uparrow}(\omega)+\tilde{\Sigma}_{\downarrow}(\omega)\right]}
\end{aligned}
$$

Before specifying the class of diagrams to be retained to approximate the dynamical self-energies $\Sigma_{\sigma}(\omega)$, the conditions necessary for Fermi-liquid behaviour to prevail at low frequencies shall be reviewed ${ }^{32}$ According to Luttinger, $\frac{45}{15}$ the imaginary part of the single selfenergy $\Sigma(\omega)$ is required to vanish as $\mathcal{O}\left(\omega^{2}\right)$ at the Fermi level $\omega=0$, which for the two self-energies $\tilde{\Sigma}_{\sigma}(\omega)=$ $\tilde{\Sigma}_{\sigma}^{\mathrm{R}}(\omega)-\mathrm{i} \operatorname{sgn}(\omega) \tilde{\Sigma}_{\sigma}^{\mathrm{I}}(\omega)$ employed by the present approach translates to the following two conditions:

$$
\begin{aligned}
& \tilde{\Sigma}_{\sigma}^{\mathrm{I}}(\omega) \sim \mathcal{O}\left(\omega^{2}\right) \quad \text { for } \omega \rightarrow 0 ; \\
& \tilde{\Sigma}_{\uparrow}^{\mathrm{R}}(\omega=0)=\tilde{\Sigma}_{\downarrow}^{\mathrm{R}}(\omega=0) .
\end{aligned}
$$

The fulfilment of the first condition shall be assumed from now on, and will be explicitly shown in Sec. IIIB for the class of diagrams chosen in the following. The second condition requires the broken symmetry $\tilde{\Sigma}_{\sigma}(\omega)$ to coincide with the fully symmetric single $\Sigma(\omega)$ at the Fermi level, thus expressing the concept of a restored spin symmetry on the lowest energy scales ${ }^{32}$ which was alluded to in the beginning of this section. Here, the additional particle-hole symmetry, $\tilde{\Sigma}_{\uparrow}(\omega)=-\tilde{\Sigma}_{\downarrow}(-\omega)$, in combination with eqs. 21), require both self-energies $\tilde{\Sigma}_{\sigma}(\omega)$ to vanish at the Fermi level. This, in turn, automatically guarantees the Friedel sum-rule pinning of the spectra, $D(\omega=0)=1 / \pi \Delta_{0}$.

In its self-energy diagrams, the LMA incorporates transverse spin fluctuations responsible for a dynamical reversion of the impurity moment. At the simplest level, to be implemented in the following, this is achieved by coupling the ladder-sum polarization propagator to the single-particle Green function,

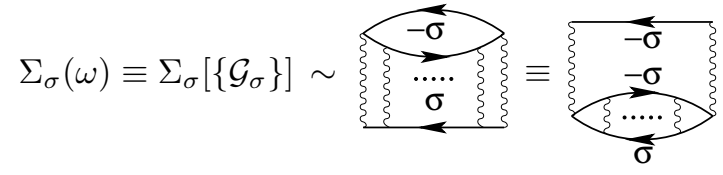

where wavy lines represent the interaction $U$ and leftgoing (right-going) solid lines stand for a UHF-like particle (hole) propagator $\mathcal{G}_{\sigma}$. Note, however, that symmetry restoration is generally not automatically guaranteed by the above class of diagrams, but can be obtained through self-consistent determination of a free parameter. In the following, the impurity moment $\mu$ will serve as such, implying that it will generally differ at self-consistency from its UHF value $\mu_{0}$ (see Sec. IIIB below).

The above approximation for the self-energies $\Sigma_{\sigma}(\omega)$ has been motivated and discussed in detail in Ref. 31, 32 34, here, its basic properties shall only be briefly reviewed: (i) in weak coupling, before the onset of moment formation, the UHF propagators $\mathcal{G}_{\sigma}$ coincide, for both $\sigma$, with the noninteracting Green functions $g$; up to (and including) second order in $U$, eq. 22 is hence equivalent to an ordinary diagrammatic perturbation expansion about the noninteracting limit. (ii) For arbitrary interaction strengths, the self-energy diagram $(22)$ describes processes where after having added at $t=0$, say, a $\sigma=\downarrow$-spin 
electron to a $-\sigma=\uparrow$-spin occupied impurity, the original $\uparrow$-spin electron hops away, thus effectively flipping the impurity spin until its return at some later time $t$ : this is precisely the dynamical spin-flip scattering, comprised in the second order of Anderson's poor man's scaling (see Ref. (9), which allows us to restore spin symmetry and to capture the strong coupling Kondo limit. (iii) In the atomic limit, where $V_{i \mathbf{k}} \equiv 0$ and therefore $\Delta_{0}=0$, the self-energy (22) vanishes, since the $-\sigma$-spin electron cannot leave the impurity at all. The LMA reduces to simple UHF, and thus becomes exact in this limit, predicting an impurity moment of $\mu_{0}=1$ and single-particle poles at $\omega= \pm U / 2$. This is salutary, since the atomic limit may be considered as an extreme of the local moment phases

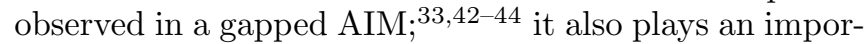
tant role in the context of the breakdown of the skeleton expansion $\frac{30}{30}$ taking place already in the metallic regime close to a Mott metal-insulator transition.25126

Before discussing the LMA impurity spectra in Sec. IV. the spin fluctuations and their dynamical coupling to the single-particle Green function will be examined in the following two paragraphs.

\section{A. Transverse spin fluctuations}

As already mentioned in the previous paragraph, one of the main ingredients for the LMA self-energy is given by dynamical spin-flip scattering processes opening the possibility to restore the spin symmetry on the lowest energy scale. Embodied in the transverse impurity-spin polarization propagators, $\Pi^{+-}(t)=\mathrm{i}\left\langle\hat{T}\left\{S_{i}^{+}(t) S_{i}^{-}(0)\right\}\right\rangle$ and $\Pi^{-+}(t)$, these scattering processes are at the simplest level accounted for by a sum of repeated particlehole interactions, as depicted by the ladder bubble in the rightmost self-energy diagram in eq. (22) which, for $\sigma=\downarrow$ $(-\sigma=\uparrow)$, leads to the familiar expression

$$
\Pi^{+-}(\omega)=\frac{{ }^{0} \Pi^{+-}(\omega)}{1-U^{0} \Pi^{+-}(\omega)},
$$

where

$$
{ }^{0} \Pi^{+-}(\omega)=\mathrm{i} \int_{-\infty}^{+\infty} \frac{\mathrm{d} \omega^{\prime}}{2 \pi} \mathcal{G}_{\downarrow}\left(\omega^{\prime}\right) \mathcal{G}_{\uparrow}\left(\omega^{\prime}-\omega\right)
$$

is the bare transverse-spin bubble. [The second polarization propagator follows by particle-hole symmetry, $\Pi^{+-}(\omega)=\Pi^{-+}(-\omega)$.]

Using the Hilbert transform relation $\sqrt{6}$ for $\mathcal{G}_{\sigma}(\omega)$ on the right-hand side (rhs) of eq. 24, yields 31

$$
\begin{aligned}
\frac{1}{\pi} \operatorname{Im}{ }^{0} \Pi^{+-}(\omega) & =\theta(\omega) \quad \int_{0}^{+|\omega|} \mathrm{d} \omega^{\prime} D_{\downarrow}^{0}\left(\omega^{\prime}\right) D_{\uparrow}^{0}\left(\omega^{\prime}-\omega\right) \\
& +\theta(-\omega) \quad \int_{-|\omega|}^{0} \mathrm{~d} \omega^{\prime} D_{\downarrow}^{0}\left(\omega^{\prime}\right) D_{\uparrow}^{0}\left(\omega^{\prime}-\omega\right) .
\end{aligned}
$$

The first term describes a spin-flipping particle-hole excitation where an electron is removed from an occupied $\uparrow$-spin state below the Fermi surface $\left(\omega^{\prime}-\omega<0\right)$ and placed into an unoccupied $\downarrow$-spin state above the Fermi surface $\left(\omega^{\prime}>0\right)$. Similarly, the second term accounts for the recombination of an $\uparrow$-spin electron into an empty $\downarrow$-spin level below the Fermi surface.

In the present AIM with a narrow host band, these processes operate mainly between the UHF orbitals, giving rise to poles in $\frac{1}{\pi} \operatorname{Im}^{0} \Pi^{+-}(\omega)$ at $\omega=2 \omega_{>}$and $\omega=-2 \omega_{<}$ with respective pole weight $q_{\gtrless}^{2}$.

For a vanishing impurity moment, as appropriate for weak coupling, the UHF levels coincide with the "molecular" orbitals of the noninteracting limit, entailing poles in $\frac{1}{\pi} \operatorname{Im}^{0} \Pi^{+-}(\omega)$ at frequencies $\omega= \pm 2 \omega_{0}$ and of spectral weight $q \simeq 1 / 4$ each. Conversely, in moderate to strong coupling, particle-hole excitations between the lower and the upper UHF Hubbard level become dominant, producing a high-frequency pole at $\omega=2 \omega_{>} \simeq U$ of weight $q_{>}^{2} \simeq 1$ in $\frac{1}{\pi} \operatorname{Im}^{0} \Pi^{+-}(\omega)$, and a corresponding lowfrequency pole at $\omega=-2 \omega_{<}$whose intensity decreases rapidly (first algebraically for $U^{\mathrm{c}} \ll U \lesssim \Delta_{0}$, where $q_{<}^{2} \sim\left[2 \omega_{0} / U\right]^{4}$, and then exponentially for $U \gg 4 \Delta_{0}$ ).

In addition to the pole contributions $\frac{1}{\pi} \operatorname{Im}^{0} \Pi^{+-}(\omega)$ contains three weak continua stemming from processes involving the narrow UHF single-particle band at the Fermi level. Two of these continua have their origin in electronic transfer between the single-particle band and one of the UHF levels; they are found at $\omega \in\left[\omega_{>}, \omega_{>}+D\right]$ and at $\omega \in\left[-\omega_{<}-D,-\omega_{<}\right]$, with respective net weight $\mathcal{O}\left(q_{\gtrless} D / \Delta_{0}\right)$, and turn out to be of little importance in the following. The third continuum appears at low frequencies, $|\omega| \leq 2 D$, and owes its existence to particle-hole excitations within the narrow UHF single-particle band itself. According to eq. 25), its behaviour in the vicinity of the Fermi level is essentially governed by the overlap of the two $D_{\sigma}^{0}(\omega)$-bands; for a metallic host, the intensity of the latter being finite at the Fermi level $\omega=0$, this results in a continuum vanishing linearly for small $\omega$,

$$
\frac{1}{\pi} \operatorname{Im}^{0} \Pi^{+-}(\omega) \stackrel{|\omega| \ll D}{\sim}|\omega| D_{\downarrow}^{0}(0) D_{\uparrow}^{0}(0)
$$

a property that is needed to prove the fulfilment of condition 21a by the present theory ${ }^{31}$ 


\section{Stability of the ladder sum propagator.}

From the Hilbert transform relation $(6)$ for $\Pi^{+-}(\omega)$, and the positivity of its imaginary part, one deduces an analyticity condition for the polarization propagator, 31

$$
\operatorname{Re} \Pi^{+-}(\omega=0)=\int_{-\infty}^{+\infty} \frac{\mathrm{d} \omega^{\prime}}{\pi} \frac{\operatorname{Im} \Pi^{+-}\left(\omega^{\prime}\right)}{\left|\omega^{\prime}\right|}>0
$$

which naturally holds for ${ }^{0} \Pi^{+-}$and the exact $\Pi^{+-}$.

Conversely, for an approximate polarization propagator, like the present ladder-sum $\Pi^{+-}$, eq. (27) is not automatically satisfied, but rather constrains the applicability of the approximation to a certain range of parameters $U$ and $\mu$.

According to eq. 23 and granted $\operatorname{Im}^{0} \Pi^{+-}(\omega=0)=$ 0 , the behaviour of $\operatorname{Re} \Pi^{+-}(\omega=0)$ is solely controlled by the bare $\operatorname{Re}^{0} \Pi^{+-}(\omega=0)$. For a vanishing impurity moment, as enforced in RHF and found self-consistently in $\mathrm{UHF}$ for $U<U_{0}^{\mathrm{c}} \equiv 4 \omega_{0}, \operatorname{Re}^{0} \Pi^{+-}(\omega=0) \simeq 1 / 4 \omega_{0}$ follows to good accuracy from the two-site approximation, implying that the corresponding ladder-sum $\Pi^{+-}$ satisfies the positivity condition (27).

Above $U_{0}^{\mathrm{c}}$, the $\mu=0$ ladder-sum $\Pi^{+-}$— which corresponds to an ordinary random phase approximation (RPA) in the transverse spin channel - becomes unstable to spin excitations since the underlying mean-field theory predicts a transition to a phase with a finite magnetic moment. A finite $\mu$, on the other hand, allows for a direct calculation of $\operatorname{Re}^{0} \Pi^{+-}(\omega=0)$ from eq. 24) by means of the identity $\mathcal{G}_{\uparrow}(\omega)-\mathcal{G}_{\downarrow}(\omega)=-U \mu \mathcal{G}_{\downarrow}(\omega) \mathcal{G}_{\uparrow}(\omega)$ and of particle-hole symmetry $D_{\sigma}^{0}(\omega)=D_{-\sigma}^{0}(-\omega)$ :

$$
U \operatorname{Re}^{0} \Pi^{+-}(\omega=0)=\frac{1}{\mu} \int_{-\infty}^{0} \mathrm{~d} \omega\left[D_{\uparrow}^{0}(\omega)-D_{\downarrow}^{0}(\omega)\right] .
$$

The latter equation shows that if the moment is determined self-consistently from the UHF propagators, $\mu=\mu_{0}$ as given in UHF by eq. (11), the rhs evaluates to unity and the corresponding ladder-sum $\Pi^{+-}$, eq. 23., has a pole at $\omega=0$. While appropriate for an insulator, where flipping the impurity spin costs no energy, in a metallic host such a spin flip is expected to be governed by the Kondo effect and thus to involve a finite energy.

In the framework of the LMA, this picture emerges naturally if $\mu$ is increased above its UHF value $\mu_{0}$, thereby shifting the pole in $\Pi^{+-}(\omega)$ from $\omega=0$ to a small but positive frequency $\omega_{\mathrm{m}}$ - closely related to the Kondo energy - and rendering $\Pi^{+-}$analytic in the sense of eq. (27).

\section{Sum rules and renormalized ladder-sum propagator.}

Any acceptable approximation for the spin-flip polarization propagator should fulfil the following sum rules:

$$
\begin{aligned}
{\left[\int_{0}^{+\infty} \pm \int_{-\infty}^{0}\right] \frac{\mathrm{d} \omega}{\pi} \operatorname{Im} \Pi^{+-}(\omega) } & =\left\langle\left[c_{i \uparrow}^{+} c_{i \downarrow}, c_{i \downarrow}^{+} c_{i \uparrow}\right]_{ \pm}\right\rangle \\
& =\left\{\begin{array}{l}
n_{i \uparrow}+n_{i \downarrow}-2\left\langle\hat{n}_{i \uparrow} \hat{n}_{i \downarrow}\right\rangle \\
\mu
\end{array}\right.
\end{aligned}
$$

which naturally hold for the noninteracting ${ }^{0} \Pi^{+-}(\omega)$, and likewise for the exact $\Pi^{+-}(\omega)$.

The second sum rule reflects the fact that, in weak coupling, $\operatorname{Im} \Pi^{+-}(\omega)$ is symmetric about the Fermi level since the impurity is found to be occupied by electrons of both spin species with equal probability; after the onset of moment formation (and before symmetry restoration), the impurity is predominantly $\uparrow$-spin occupied and spectral intensity at positive energies, associated with processes flipping the impurity spin from $\uparrow$ to $\downarrow$, should dominate.

As for the first sum rule, its rhs is comprised between $1 / 2$ and 1 for the particle-hole symmetric AIM, since the expectation to find a doubly occupied impurity $\left\langle\hat{n}_{i \uparrow} \hat{n}_{i \downarrow}\right\rangle$ varies from $1 / 4$ in weak to 0 in strong coupling and, in any case, $n_{i \uparrow}+n_{i \downarrow}=1$; both limits are captured by the approximate expression $\frac{1}{2}\left[1+\mu^{2}\right]$ which follows by recasting the double occupancy as $\left\langle\hat{n}_{i \uparrow} \hat{n}_{i \downarrow}\right\rangle=\frac{1}{4}\left(\left\langle\hat{n}_{i}^{2}\right\rangle-\right.$ $\left.\left\langle\hat{\mu}_{i}^{2}\right\rangle\right)$ and subsequently factorizing the quartic Fermion operators via a Hartree-Fock decoupling.

For the present narrow-band AIM, the above sum rules are naturally fulfilled in weak coupling, where $\Pi^{+-}(\omega)$ reduces essentially to the noninteracting ${ }^{0} \Pi^{+-}(\omega)$. More surprisingly, also in the strong coupling (Kondo) regime, $U \gg 4 \Delta_{0}$, both sum rules are found to be approximately satisfied for values of the magnetic moment required by symmetry restoration.

Problems are found to arise primarily for moderate interactions, large enough to find the impurity moment well established, but far from the Kondo limit. For this range of interactions, the main features of the ladder-sum polarization propagator $\Pi^{+-}(\omega)$ are accurately described in the two-site approximation; it reduces to two poles, with frequencies and weights given analytically by

$$
\begin{aligned}
& \omega_{\mathrm{m}}^{ \pm}=2 \omega_{0}\left[\gamma y \pm \sqrt{(\gamma y)^{2}+\gamma}\right], \\
& q_{\mathrm{m}}^{ \pm}=\frac{\left[\gamma y \pm \sqrt{(\gamma y)^{2}+\gamma}\right]^{2}}{4 \gamma \sqrt{\left[y^{2}+1\right]\left[(\gamma y)^{2}+\gamma\right]}},
\end{aligned}
$$

where $y=\mu U / U_{0}^{\mathrm{c}}$ and $\gamma=1-\frac{U / U_{0}^{\mathrm{c}}}{\sqrt{y^{2}+1}}$. Incidentally, eqs. (30) encompass the bare (broken symmetry) ${ }^{0} \Pi^{+-}(\omega)$, which follows for $\gamma=1$, as well as the noninteracting polarization bubble, obtained by setting $U=0$ and $\gamma=1$. 
Within the two-site approximation, appreciable sumrule violations occur for $U \gg U_{0}^{\mathrm{c}}$, where the stability criterion (27) and the definition of the magnetic moment itself constrain $\mu$ to the narrow interval between the UHF moment $\mu_{0}$ and 1 . In this regime, the deviation of the moment from its UHF value defines a small positive parameter $\delta=\mu-\mu_{0}$. The position and weights of the ladder-sum poles, eqs. 30 , can then be expanded in terms of the square root of this parameter which, using $\gamma \simeq \mu_{0} \delta+\mathcal{O}\left(\delta^{2}\right)$, yields

$$
\begin{aligned}
\omega_{\mathrm{m}}^{ \pm} & \simeq \pm 2 \omega_{0} \sqrt{\mu_{0} \delta}+\frac{U}{2} \mu_{0}^{2} \delta+\mathcal{O}\left(\sqrt{\delta}^{3}\right) \\
q_{\mathrm{m}}^{ \pm} & \simeq \frac{\omega_{0}}{U \sqrt{\mu_{0} \delta}} \pm \frac{\mu_{0}}{2}+\mathcal{O}(\sqrt{\delta}) .
\end{aligned}
$$

By consequence, the integrals in eq. 290 evaluate to $q_{\mathrm{m}}^{+}+$ $q_{\mathrm{m}}^{-}=2 \omega_{0} / U \sqrt{\mu_{0} \delta}+\mathcal{O}(\sqrt{\delta})$ and $q_{\mathrm{m}}^{+}-q_{\mathrm{m}}^{-}=\mu_{0}$; the $1 / \sqrt{\delta}$ singularity of the first result indicates a strong violation of the first sum rule (while the second is found to be fulfilled to leading order even in this regime).

Within the present approach, this problem can be overcome by renormalization of the ladder-sum propagator, i.e., by multiplication of $\operatorname{Im} \Pi^{+-}(\omega)$ with two different constants above and below the Fermi level, $\omega=0$, chosen to comply with both sum rules (29). [The corresponding real part can subsequently be calculated from the Hilbert transform (6).] In the two-site approximation, the renormalized weights - which have to be used instead of eq. (30b) — are explicitly given by

$$
q_{\mathrm{m}}^{ \pm}=\frac{1}{4}(1 \pm \mu)^{2} .
$$

Henceforth, unless explicitly stated otherwise, $\Pi^{+-}(\omega)$ will always stand for such a renormalized spin-flip ladder sum.

\section{The full spin-flip polarization propagator.}

Beyond the two-site approximation, where the polarization propagator solely consists of two poles, with frequencies given by eq. (30a) and renormalized weights (32), the full ladder-sum $\Pi^{+-}$contains the following additional features:

(i) The full $\Pi^{+-}(\omega)$ inherits its continua from the bare ${ }^{0} \Pi^{+-}(\omega)$; these are located at frequencies $\omega \in$ $\pm\left[\omega_{\gtrless}, \omega_{\gtrless}+D\right]$ and $|\omega| \leq 2 D$.

(ii) For moments $\mu$ which only exceed the corresponding UHF moment very slightly, as appropriate in strong coupling, $U \gg 4 \Delta_{0}$, the pole frequencies (30a) may lie within the low-energy continuum $|\omega| \leq 2 \bar{D}$; instead of poles, the full $\Pi^{+-}(\omega)$ will thus have sharp resonances at these frequencies.

(iii) In addition to the above mentioned poles, the full $\Pi^{+-}(\omega)$ shows a third collective pole, induced by the RPA-like structure of eq. (23) and the logarithmic singularity of $\operatorname{Re}^{0} \Pi^{+-}(\omega)$ at the upper edge of the polarization band found for $\omega \in\left[-\omega_{<}-D,-\omega_{<}\right]$; of tiny weight throughout the whole range of interactions, this pole can be safely neglected in practice.

\section{B. The LMA self-energy}

For a $\downarrow$-spin electron, the self-energy diagram 22 translates to

$$
\Sigma_{\downarrow}(\omega)=U^{2} \int_{-\infty}^{+\infty} \frac{\mathrm{d} \omega^{\prime}}{2 \pi \mathrm{i}} \Pi^{+-}\left(\omega^{\prime}\right) \mathcal{G}_{\uparrow}\left(\omega-\omega^{\prime}\right) ;
$$

and the $\uparrow$-spin self-energy follows by particle-hole symmetry $\Sigma_{\uparrow}(\omega)=-\Sigma_{\downarrow}(-\omega)$.

In analogy to the calculus of the spin-flip polarization propagator, useful expressions for the self-energy $\Sigma_{\downarrow}(\omega)=\Sigma_{\downarrow}^{\mathrm{R}}(\omega)-\mathrm{i} \operatorname{sgn}(\omega) \Sigma_{\downarrow}^{\mathrm{I}}(\omega)$ are obtained by inserting the Hilbert transforms for the broken-symmetry $\Pi^{+-}$ and $\mathcal{G}_{\uparrow}$ in the integrands on the rhs of eq. (33), yielding for the imaginary part

$$
\begin{gathered}
\Sigma_{\downarrow}^{\mathrm{I}}(\omega)=\theta(\omega) U^{2} \int_{0}^{+|\omega|} \mathrm{d} \omega^{\prime} \operatorname{Im} \Pi^{+-}\left(\omega^{\prime}\right) D_{\uparrow}^{0}\left(\omega-\omega^{\prime}\right) \\
+\theta(-\omega) U^{2} \int_{-|\omega|}^{0} \mathrm{~d} \omega^{\prime} \operatorname{Im} \Pi^{+-}\left(\omega^{\prime}\right) D_{\uparrow}^{0}\left(\omega-\omega^{\prime}\right) ;
\end{gathered}
$$

and a corresponding real part, $\Sigma_{\downarrow}^{\mathrm{R}}(\omega)$, which follows by Hilbert transform.

The evaluation of the integrals in eq. (34) is greatly facilitated by the structure of the polarization propagator $\Pi^{+-}(\omega)$, whose spectral intensity resides - as pointed out in Sec. III A - throughout the entire range of interactions almost exclusively in two sharp low-energy modes at $\omega=\omega_{\mathrm{m}}^{ \pm}$. Except in strong coupling, $U \gg 4 \Delta_{0}$, the dominant contribution to the self-energy arises by coupling these modes to the UHF $\uparrow$-spin orbitals, resulting in two poles or sharp resonances in $\Sigma_{\downarrow}^{\mathrm{I}}(\omega)$, the first occurring at $\omega=\omega_{\mathrm{m}}^{+}+\omega_{<}>0$ (net weight $U^{2} q_{\mathrm{m}}^{+} q_{<}$), and the second at $\omega=\omega_{\mathrm{m}}^{-}-\omega_{>}<0$ (net weight $U^{2} q_{\mathrm{m}}^{-} q_{>}$).

The self-energy pole at positive frequencies is associated with single-particle excitations where an extra $\downarrow$ spin electron is introduced on the impurity, and the initial $\uparrow$-spin impurity electron hops off to the host. The same result could have been obtained from the original groundstate by placing the extra electron directly in the empty $\uparrow$-spin UHF orbital (energy cost $\omega_{<}$) and simultaneously flipping the impurity moment from $\uparrow$ to $\downarrow$ (energy cost $\left.\omega_{\mathrm{m}}^{+}\right)$. Analogously, the $\Sigma_{\downarrow}^{\mathrm{I}}$-pole at negative frequencies is caused by a $\downarrow$-spin electron being taken off the impurity and subsequently replaced by an $\uparrow$-spin electron from the host, yielding an $\uparrow$-spin electron in the UHF orbital below the Fermi level (energy gain $\omega_{>}$) and a flip of the impurity moment from $\downarrow$ to $\uparrow$ (energy gain $\left.\left|\omega_{\mathrm{m}}^{-}\right|\right)$. As this requires the impurity to be initially $\downarrow$-spin occupied, the 
latter self-energy contribution will decline as the impurity moment $\mu$ approaches saturation.

In addition to these collective modes, eq. (34) predicts several sets of narrow self-energy continua that arise from band contributions in $D_{\uparrow}^{0}$ and $\Pi^{+-}$; such continua occur for $\omega \geq \omega_{\mathrm{m}}^{+}$, for $\omega \leq \omega_{\mathrm{m}}^{-}$and for $|\omega| \geq \omega_{>}+\omega_{<}$(all of width $D$ ), for $|\omega| \geq \omega_{\gtrless}$ (width $2 D$ ), and for $|\omega| \leq 3 D$. All these continua remain generally weak over the whole range of parameters, albeit with one exception: as the impurity moment $\mu$ approaches its UHF value from above, $\mu \rightarrow \mu_{0}+0$, and consequently $\omega_{\mathrm{m}}^{ \pm} \rightarrow 0-$ a situation that becomes relevant in the strong coupling Kondo limit discussed in Sec. III B 2 below - the minor UHF singleparticle pole vanishes exponentially [see eq. (15b)], and the dominant self-energy contribution is transferred to the low-energy continuum located at $|\omega| \leq 3 D$.

That the low-frequency behaviour of the latter continuum fulfils the first condition for symmetry restoration, eq. 21a), may be seen as follows: the continuum is generated, as in eq. (34), by convoluting the UHF band for $\uparrow$-spin electrons with the low-frequency continuum of $\mathrm{Im} \Pi^{+-}$; the latter vanishes linearly in $\omega$ as $\omega \rightarrow 0$ [a consequence of eq. $(26)$ in combination with the analyticity condition (27), which for a ladder-sum propagator implies $1-U \operatorname{Re}^{0} \Pi^{+-}(\omega=0)>0$ ], entailing their convolution to behave as $\left[\tilde{\Sigma}_{\downarrow}^{\mathrm{I}}(\omega) \equiv\right] \Sigma_{\downarrow}^{\mathrm{I}}(\omega) \propto \omega^{2}$ for $|\omega| \ll D$.

The second condition, eq. 21b, by contrast, is not automatically fulfilled by the present theory. As pointed out before, it reduces under particle-hole symmetry to $\tilde{\Sigma}_{\downarrow}^{\mathrm{R}}(\omega=0)=\Sigma_{\downarrow}^{\text {stat }}+\Sigma_{\downarrow}^{\mathrm{R}}(\omega)=0$, which, under the assumption $\Sigma_{\downarrow}^{\text {stat }} \simeq U \mu / 2$, becomes

$$
\Sigma_{\downarrow}^{\mathrm{R}}(\omega=0)+\frac{U}{2} \mu=0 .
$$

Within the LMA, this condition will be satisfied by tuning the impurity moment $\mu$ within the interval $\mu_{0}<$ $\mu \leq 1$, prescribed by the analyticity condition 27). Despite the generally narrow range of possible values, a solution can always be found since the dynamical contribution, $\Sigma_{\downarrow}^{\mathrm{R}}(\omega=0)$, is very sensitive to the exact position of the majority self-energy pole and hence implicitly to the spin-flip scale $\omega_{\mathrm{m}}^{+}$. The latter quantity is highly responsive to changes of the impurity moment and thus effectively controls the condition 35 . In a more general perspective, $\omega_{\mathrm{m}}^{+}$— which vanishes for an ordinary UHF ground-state with finite impurity moment (see Sec. III A 1) - may be considered as a new order parameter that, for a state with local moments, determines whether Kondo physics takes place or not.

The above analysis is corroborated by the numerical results displayed in Fig. 1, confirming that, for a wide range of interactions, $U \gg U_{0}^{\mathrm{c}}$, the impurity moment $\mu$ needed to comply with condition 35 is indeed very close to the UHF moment $\mu_{0}$.

In the remaining two paragraphs of this section, condition (35) in combination with the LMA self-energy will be studied in two regimes for which analytic results can be worked out: (i) the two-site limit, appropriate for weak to moderate interactions; and (ii) the strong coupling Kondo limit.

\section{The self-energy in the two-site limit.}

The two-site approximation $-D \rightarrow 0$ albeit with finite $\omega_{0} \simeq \sqrt{2 \Delta_{0} D / \pi}$ - allows for the LMA self-energy to be written in simple analytical terms and, more importantly, suggests a two-pole structure for $\Sigma_{\sigma}(\omega)$ that, for $\omega \gg$ $|D|$ and up to moderate interactions $U \lesssim \Delta_{0}$, correctly reproduces the main results of a complete version of the LMA for the present narrow-band AIM.

In weak coupling, $U \ll U_{0}^{\mathrm{c}}$, UHF predicts a solution with a vanishing impurity moment, $\mu_{0}=0$, and singleparticle propagators which, independent of spin and impurity type, reduce to the noninteracting Green function, $\mathcal{G}_{\sigma}(\omega) \equiv g(\omega)$. The corresponding self-energies $\Sigma_{\sigma}(\omega)$ are hence odd functions of $\omega$ and their real parts satisfy the condition (35) by symmetry. As obvious from eq. (22), the leading contribution to the self-energy diagrams stems from ordinary $2 \mathrm{PT}$ about the noninteracting ground state and is hence overwhelmingly dominated by poles at $\omega \simeq \pm 3 \omega_{0}$ of weight $Q \sim U^{2} / 8$ each.

Above some critical interaction $U^{\mathrm{c}}$ - which is of the same order of magnitude but slightly smaller than the corresponding UHF critical interaction $U_{0}^{\mathrm{c}}$ - the nonmagnetic solution becomes unstable and a finite moment forms on the impurity. As illustrated in Fig. 1 , the LMA moment $\mu$ saturates rapidly with increasing $U$, and its numerical values fit accurately to a square-root law [which, in contrast to and despite the similarity with eq. 13, is not even exact in the two-site limit]:

$$
\mu \simeq \begin{cases}\sqrt{1-\left(U^{\mathrm{c}} / U\right)^{2}} & \text { for } U>U^{\mathrm{c}}:=2 \sqrt{2} \omega_{0} \\ 0 & \text { for } U<U^{\mathrm{c}}\end{cases}
$$

As pointed out above, symmetry restoration and consequently the Fermi-liquid nature of the single-particle excitations hinge, in this regime, almost exclusively on the energy cost $\omega_{\mathrm{m}}$ for flipping the impurity moment. For simplicity, in what follows, the latter quantity will be thought of as an independent parameter, to be determined from condition (35), and the impurity moment will be kept at its UHF value $\mu_{0}$ instead.

Under these assumptions (which are in concord to leading-order with the analysis in Sec. III A 2), $\Pi^{+-}$ is dominated by poles at $\omega_{\mathrm{m}}^{ \pm}= \pm \omega_{\mathrm{m}}$, of renormalized weights $q_{\mathrm{m}}^{ \pm}=\frac{1}{4}\left(1 \pm \mu_{0}\right)^{2}$, and $\Sigma_{\downarrow}(\omega)$ is constituted by a majority pole at $\omega=\omega_{\mathrm{m}}+\omega_{<}$(net weight $\left.U^{2} q_{\mathrm{m}}^{+} q_{<} \equiv 4 \omega_{0}^{2} q_{>}\right)$and a minority pole at $\omega=-\omega_{\mathrm{m}}-\omega_{>}$ (net weight $U^{2} q_{\mathrm{m}}^{-} q_{>} \equiv 4 \omega_{0}^{2} q_{<}$). The magnetic energy scale follows finally from eq. (35), viz.

$$
\omega_{\mathrm{m}}=-\frac{U}{4}+J+\sqrt{\left(\frac{U}{4}\right)^{2}+J^{2}+\omega_{0}^{2}},
$$


where, again, $J \equiv 4 \omega_{0}^{2} / U$ stands for the antiferromagnetic exchange coupling constant.

At the critical interaction for moment formation in UHF, where $U=U_{0}^{\mathrm{c}}$ and $U / 4=J=\omega_{0}$, the magnetic energy starts out at $\omega_{\mathrm{m}}=\sqrt{3} \omega_{0}$, and then decreases as $\omega_{\mathrm{m}} \sim 3 J / 2$ for interactions $U \gg U_{0}^{\mathrm{c}}$ (the relevance of the two-site approximation being subject to $\omega_{\mathrm{m}} \gg D$, or equivalently $U \lesssim \Delta_{0}$ as previously).

Due to the simplicity of the two-site approximation, two further virtues of the LMA become manifest: first, the renormalization procedure of $\Pi^{+-}(\omega)$ - which enforces the sum rules (29) - guarantees the LMA self-energy (33) to fulfil an analogous set of sum rules for the interaction self-energy $\tilde{\Sigma}_{\downarrow}(\omega) \equiv$ $U^{2}\left\langle\left\langle c_{i \downarrow} \delta \hat{n}_{i \uparrow} ; \delta \hat{n}_{i \uparrow} c_{i \downarrow}^{+}\right\rangle\right\rangle_{\omega}$,

$$
\begin{aligned}
{\left[\int_{0}^{+\infty} \pm \int_{-\infty}^{0}\right] \frac{\mathrm{d} \omega}{\pi} \tilde{\Sigma}_{\downarrow}^{\mathrm{I}}(\omega) } & =U^{2}\left\langle\left[c_{i \downarrow} \delta \hat{n}_{i \uparrow}, \delta \hat{n}_{i \uparrow} c_{i \downarrow}^{+}\right]_{ \pm}\right\rangle \\
& \simeq \frac{U^{2}}{4}\left(1-\mu_{0}^{2}\right) \times\left\{\begin{array}{l}
1 \\
\mu_{0}
\end{array}\right.
\end{aligned}
$$

the sole condition being that the many-body expectation values on the rhs are evaluated by a Hartree-Fock factorization, as shown on the second line of eq. 38) (which, again, correctly captures the limits of weak and strong coupling).

The second virtue concerns the conventional single selfenergy $\Sigma$ : in Lanczos calculations by Hofstetter and Kehrein, 25 this $\Sigma(\omega)$ is found to have poles on an energy scale $\omega \sim \sqrt{\Delta_{0} D}$ which cannot be explained in any order of the skeleton expansion. ${ }^{25 \mid 26}$ Moreover, these poles have been shown to depend little or not at all on the interaction strength, 30 occurring in the limits of strong and weak coupling exactly at $\omega= \pm 3 \omega_{0}$, with net spectral weight $Q \sim U^{2} / 8$ each. Responsible in the atomic limit $V_{i \mathbf{k}} \sim \omega_{0}=0$ for the $\Sigma(\omega) \sim 1 / \omega$ characteristics of the insulator, they are an intrinsic feature of the narrowband AIM.44

It has been argued in the beginning of this paragraph that, in weak coupling $\left(U \leq U^{\mathrm{c}}\right)$, both LMA self-energies coincide and inherit their main properties from $2 \mathrm{PT}$, whose poles in turn precisely respect the desired properties. But also for $U \gg U^{\mathrm{c}}$, where the impurity moment saturates and $\omega_{\mathrm{m}} \simeq \frac{3}{2} J$, such poles occur on a similar energy scale in the symmetry-restored single LMA selfenergy, $\Sigma(\omega)$. The latter depends, as in eq. (20), on the self-energies $\tilde{\Sigma}_{\sigma}$ which in the limit $U \gg U^{\text {c }}$ reduce to

$$
\tilde{\Sigma}_{\downarrow}(\omega) \simeq \frac{\frac{U}{2} \omega\left[\omega+\frac{U}{2}\right]}{\omega^{2}+\frac{U}{2} \omega-4 \omega_{0}^{2}}
$$

and $\tilde{\Sigma}_{\uparrow}(\omega)=-\tilde{\Sigma}_{\downarrow}(-\omega)$; insertion of which into eq. 20 generates a single self-energy constituted, to leading order in $J \sim 1 / U$, by poles at $\omega \simeq \pm \sqrt{5} \omega_{0}$ of spectral weight $Q \sim U^{2} / 8$ each. Hence apart from the pole frequency prefactor $(\sqrt{5} \simeq 2.23$ instead of 3 ), the LMA self-energy matches the above mentioned properties of the exact solution also for $U \gg U^{\mathrm{c}}$. The discrepancy in the prefactor is possibly due to the inability of the twosite approximation to describe the true strong coupling regime, $U \gg 4 \Delta_{0}$.

\section{The self-energy in the Kondo limit.}

In genuinely strong coupling, $U \gg 4 \Delta_{0}$, the polarization propagator $\Pi^{+-}(\omega)$ consists in essence of a single sharp resonance peaked at a frequency $\omega_{\mathrm{m}}$ which is tiny compared to any other frequency scale involved in the problem, even $D$. The corresponding self-energy, obtained by convoluting this resonance with the singleparticle UHF propagator as in eq. (34), is thus governed by resonant spin-flip scattering within the metallic singleparticle band at the Fermi level itself, i.e., the Kondo effect, whereas the orbital contributions, predominant in the two-site limit, vanish exponentially [see eq. (15b)].

Under these circumstances, and granted $\mu_{0} \simeq 1$, condition (35) can again be solved analytically, yielding an exponentially small spin-flip energy, $\omega_{\mathrm{m}} \simeq$ $D \exp \left[-\pi U / 8 \Delta_{0}\right]$, which - apart from the prefactor concurs with the LMA results for the AIM with a flat and infinite wide hybridization band ${ }^{[31}$ Far from the band edges of the low-energy continuum, the interaction selfenergy can be written as a function of a single parameter, $\tilde{\omega}=\omega / \omega_{\mathrm{m}}$, viz.

$$
\tilde{\Sigma}_{\downarrow}(\omega) \stackrel{|\omega| \ll D}{\sim} 4 \Delta_{0}\left[\frac{1}{\pi} \ln |1-\tilde{\omega}|-\mathrm{i} \theta(\tilde{\omega}-1)\right],
$$

and is otherwise independent of the original parameters $U$ and $D$.

This low-fequency scaling behaviour of the self-energy - which is ultimately responsible for the scaling properties of the single-particle resonance - is a hallmark of AIMs with metallic hosts.19/31/32 The associated prediction of an exponentially small magnetic energy scale, closely related to the Kondo temperature, emerges similarly from Anderson's poor man's scaling for the $s$-d model, and from a strong-coupling expansion of the exact Bethe ansatz solution for the AIM.10

\section{SPECTRAL EVOLUTION IN THE NARROW-BAND REGIME.}

In this section, the numerically obtained LMA impurity spectra $D(\omega)=-\frac{1}{\pi} \operatorname{sgn}(\omega) \operatorname{Im} G(\omega)$ [with $G(\omega)$ the spin-symmetric single-particle Green function of eq. (16b)] will be presented next to corresponding UHF and $2 \mathrm{PT}$ results. The section also comprises a comparison of the LMA spectra with Lanczos calculations performed by Hofstetter and Kehrein 25 for an $11+1$-site Anderson star with host bandwidth $D=10^{-4} \Delta_{0}$. 
Further analytic rationales reveal how the spectrum evolves with interaction strength and suggest an interpretation of its main features in simple physical terms: for weak to moderate interactions, $U \lesssim \Delta_{0}$, the reasoning is based on the two-site approximation where the host band is taken to be infinitely narrow; conversely, in strong coupling, $U \gg 4 \Delta_{0}$, similarities with the opposite case of an infinitely wide host band emerge in the immediate vicinity of the Fermi level, and ultimately lead to the characteristic scaling behaviour of the Kondo resonance.19131

\section{A. Weak coupling: $U \ll U^{\mathrm{c}}$}

For $U$ much smaller than the critical interaction for moment formation, $U^{\mathrm{c}}$, the LMA connects smoothly to 2PT (see Sec. III B 1), which in turn exactly captures the two-site limit for any interaction strength. By analogy to the latter, extensively discussed in Ref. 30, the LMA single-particle spectrum (depicted in the last graph of Fig. 33 is overwhelmingly dominated by the "molecular orbitals," occurring at minimally lower frequencies $|\omega| \lesssim$ $\omega_{0}$ than in the noninteracting limit. Additionally, for any nonzero $U$, a pair of weak poles arises at $\omega \simeq \pm 3 \omega_{0}$ : these are the precursors of the Hubbard satellites, and start out with net spectral weight $q \sim \mathcal{O}\left(\left[U / \omega_{0}\right]^{2}\right)$ each.
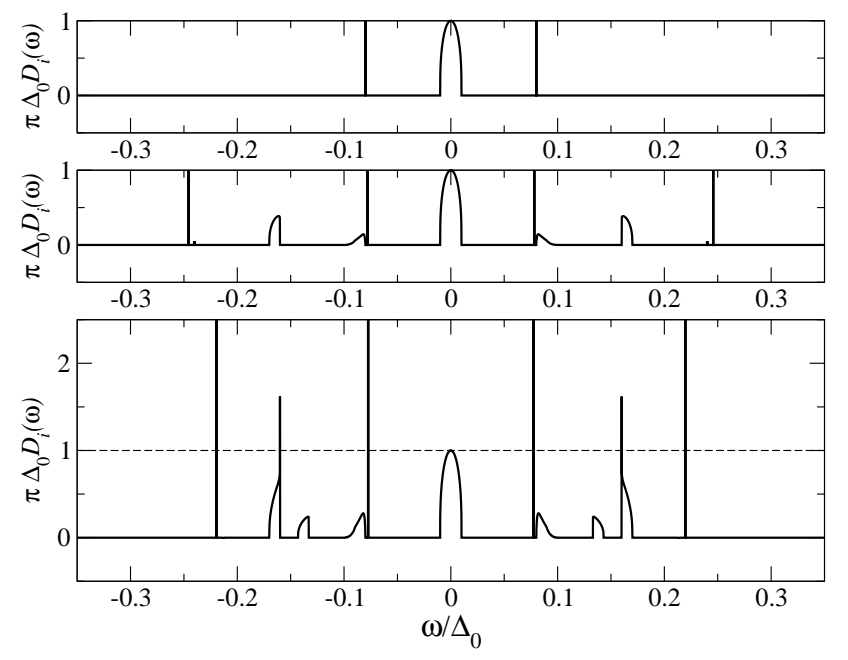

FIG. 3: UHF (top), 2PT (middle), and LMA (bottom) impurity spectra, $\left.\pi \Delta_{0} D_{(} \omega\right)$ vs. $\omega / \Delta_{0}$, for bandwidth $D=0.01 \Delta_{0}$, i.e., $\omega_{0} \simeq 0.08 \Delta_{0}$ and $U^{\mathrm{c}} \simeq 0.23 \Delta_{0}$, and interaction strength $U=0.1 \Delta_{0}$. Discrete levels are represented by vertical lines. The Fermi energy is $\omega=0$.

Fig. 3 depicts the impurity spectra for $D=0.01 \Delta_{0}$ and $U=0.1 \Delta_{0}$, i.e., an interaction strength that is weak compared to $\Delta_{0}$, but already appreciable with respect to $U^{\mathrm{c}} \simeq 2 \sqrt{2} \omega_{0} \simeq 0.23 \Delta_{0}$ ( or $U_{0}^{\mathrm{c}} \equiv 4 \omega_{0} \simeq 0.32 \Delta_{0}$ ). Since $U<U^{\mathrm{c}}$, the LMA converges to a solution without magnetic moment, and the numerically computed LMA spectra (bottom panel) carry mainly the signature of $2 \mathrm{PT}$ (middle panel), namely: (i) the "molecular orbitals", occurring at $\omega \simeq \pm 0.077 \Delta_{0}$, dominate the spectrum and carry in total more than $92 \%$ of the intensity; (ii) the outer set of poles, at $\omega \simeq \pm 0.22 \Delta_{0}$, carrying approximately $6.6 \%$ of the total spectral weight, occur slightly inside their $2 \mathrm{PT}$ counterparts at $\omega \simeq \pm 0.25 \Delta_{0}$; (iii) various weak band contributions which (with one exception) also bear great similarity with $2 \mathrm{PT}$, the most prominent being the essentially unrenormalized Fermi-liquid continuum of width $2 D$ and net spectral weight $\mathcal{O}\left(D / \Delta_{0}\right)$ around the Fermi level. These results clearly contrast with UHF, in the top panel of Fig. 3, whose spectra, for the reasons already pointed out in Sec. IIB, coincide with the noninteracting limit.

\section{B. Moderate coupling: $U^{\mathrm{c}} \ll U \lesssim \Delta_{0}$}

In the regime of moderate interaction strengths - defined by $U^{\mathrm{c}} \ll U \lesssim \Delta_{0}$ or, equivalently, $D \ll J \ll \omega_{0}$ a well-established local moment resides on the impurity. The energy cost $\omega_{\mathrm{m}} \simeq \frac{3}{2} J$ for flipping this moment being large compared to the host bandwidth $D$, the two-site scenario of Sec. IIIB 1 remains appropriate - except, of course, within the low-energy Fermi-liquid continuum $|\omega|<D$ itself.

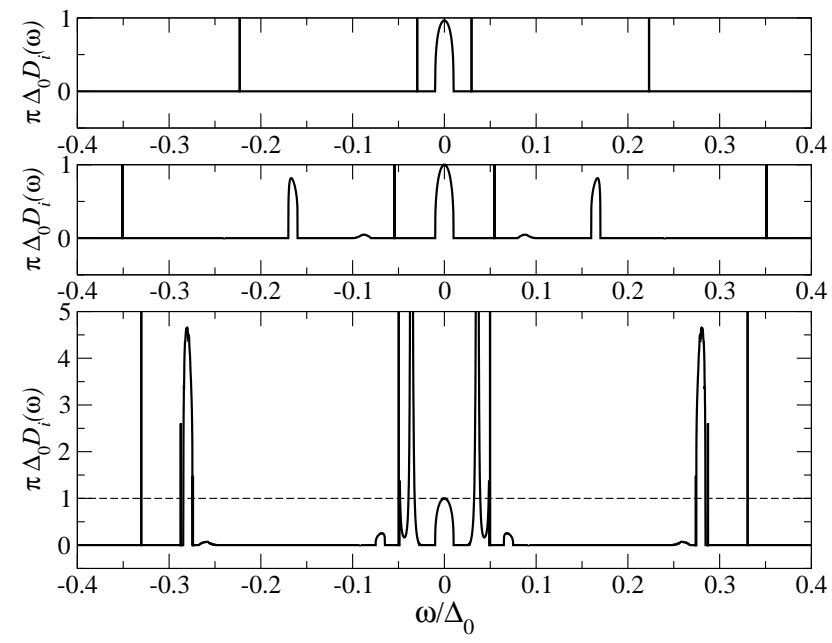

FIG. 4: UHF (top), 2PT (middle), and LMA (bottom) impurity spectra, $\left.\pi \Delta_{0} D_{(} \omega\right)$ vs. $\omega / \Delta_{0}$, for bandwidth $D=0.01 \Delta_{0}$, i.e., $\omega_{0} \simeq 0.08 \Delta_{0}$ and $U^{\mathrm{c}} \simeq 0.23 \Delta_{0}$, and interaction strength $U=0.5 \Delta_{0}\left(J \simeq 0.05 \Delta_{0}\right)$. Discrete levels are represented by vertical lines. The Fermi energy is $\omega=0$.

For a host of bandwidth $D=0.01 \Delta_{0}$, chosen in Fig. 4 for clear resolution on all relevant energy scales, the above conditions of moderate interaction strengths are never truly met, since $U^{\mathrm{c}} \simeq 0.23 \Delta_{0}$ and $\Delta_{0}$ are of similar orders of magnitude. Nevertheless, for $U=0.5 \Delta_{0}$ (as in the figure), where the LMA impurity moment is well established $\left(\mu \simeq 0.89\right.$ vs. $\mu_{0} \simeq 0.78$ for UHF, see Fig. 1), the moderate coupling regime of the two- 
site approximation is a good starting point for the analysis of the impurity spectra. Their most prominent feature, accounting for approximately $68 \%$ of the spectral intensity, are the high-energy Hubbard satellites at $|\omega| \simeq \frac{1}{2} U+\frac{3}{2} J \simeq 0.33 \Delta_{0}$. They are found to occur remarkably close to their $2 \mathrm{PT}$ counterparts at $|\omega| \simeq 0.35 \Delta_{0}$ (expected in the two-site limit of $2 \mathrm{PT}$ at $|\omega| \simeq \frac{1}{2} U+\frac{5}{2} J \simeq$ $\left.0.38 \Delta_{0}\right)$, whereas the UHF correction to the atomic limit (where $|\omega| \equiv \frac{U}{2}$ ) operates in the opposite direction, $|\omega| \simeq \frac{1}{2} U-\frac{1}{2} J \simeq 0.22 \Delta_{0}$. A new feature on the high energy scale are the side bands for $|\omega| \geq \omega_{>}+\omega_{<} \simeq 0.27 \Delta_{0}$ (and, very faint, for $|\omega| \geq \omega_{>} \simeq 0.25 \Delta_{0}$ ), stemming from many-body self-energy continua (see Sec. IIIB): strongly enhanced with respect to their $2 \mathrm{PT}$ progenitors (located in the middle panel at $|\omega|=2 \omega_{0} \simeq 0.16 \Delta_{0}$ and $\left.\omega_{0} \simeq 0.08 \Delta_{0}\right)$, these side bands will, for larger $U$, eventually merge with the Hubbard levels, thereby destroying their discrete nature. This, in turn, may be viewed as an extreme version of the many-body broadening effect generic to the AIM and likewise observed for hosts of large bandwidth. 3132

In the low-energy sector - governed by the antiferromagnetic scale $J=4 \omega_{0}^{2} / U \simeq 0.05 \Delta_{0}$ and the bandwidth $D=0.01 \Delta_{0}$ - the LMA impurity spectra reveal a much richer structure than their UHF or 2PT counterparts. While all three approaches predict the low-energy continuum, ranging from $\omega=-D$ to $D$ and accounting for $0.4 \%$ of the total spectral intensity, to suffer very little renormalization with respect to the noninteracting limit, the spectra differ significantly on the $J$-scale, where simpler UHF and $2 \mathrm{PT}$ both predict a single pair of discrete levels (which can be viewed as remnants of the molecular orbitals). An additional, second pair of levels emerges in the LMA already in the framework of the two-site approximation: the pole equation for the $\downarrow$-spin electron, $\omega-\Delta_{\mathrm{R}}(\omega)-\Sigma_{\downarrow}(\omega)=0$, with $\Sigma_{\downarrow}(\omega)$ from eq. 39, yields the upper Hubbard satellite together with two low-energy poles at, to leading order in $J$,

$$
\omega_{1,2} \simeq-\frac{1 \pm \sqrt{17}}{4} \mathrm{~J},
$$

while, the $\uparrow$-spin pole equation, leads to the lower Hubbard satellite and poles at $\omega=-\omega_{1,2}$.

The low-energy poles at $\omega= \pm \omega_{1,2}$ can be interpreted in the following way: the outer levels at $|\omega|=\left|\omega_{1}\right| \simeq$ $1.28 J \simeq 0.065 \Delta_{0}$ (of net weight $\left.q_{1} \simeq 0.51\left[\mathrm{~J} / \omega_{0}\right]^{2}\right)$, result from a shift of the UHF orbital remnants, initially found at $|\omega|=\omega_{<} \simeq \frac{1}{2} J$, and occur remarkably close to their $2 \mathrm{PT}$ counterparts at $|\omega| \simeq \frac{3}{2} J \simeq 0.076 \Delta_{0}$; the inner poles, at frequencies $|\omega|=\omega_{2} \simeq 0.78 J \simeq 0.04 \Delta_{0}$ and of net weight $q_{2} \simeq 0.12\left[\mathrm{~J} / \omega_{0}\right]^{2}$, are entirely new features, which owe their existence to resonant spin-flip scattering as accounted for by the majority self-energy pole at $\omega=\omega_{<}+\omega_{\mathrm{m}} \simeq 2 J$. In UHF, this pole is naturally absent due to the total lack of dynamics; but it also misses in 2PT, albeit for subtler reasons: assuming a perfect parity between host and impurity, as appropriate for an actual two-site problem, a resonance process that tends to localize a spin flip on the impurity rather than on the host is ruled out in 2PT by construction.

Despite the rather poor concord with the definition of moderate coupling, the numerically calculated LMA spectrum of Fig. 4 corroborates the above analysis to reasonable accuracy, displaying in the low-energy sector a four-peak structure with (i) levels at $|\omega| \simeq 0.05 \Delta_{0} \simeq J$ (instead of the expected $1.28 J$ ) and carrying about $21 \%$ of the spectral intensity; and (ii) sharply peaked continua at $|\omega| \simeq 0.7 \mathrm{~J} \simeq 0.035 \Delta_{0}$, accounting for roughly $8 \%$ of the spectral weight, stemming from the merger of the above mentioned inner poles, expected at $\omega \simeq \pm 0.78 \mathrm{~J}$, with self-energy bands.

\section{Comparison with Lanczos spectra.}

In their article, ${ }^{25}$ Hofstetter and Kehrein present lowfrequency Lanczos spectra for an Anderson impurity coupled to a host of 11 sites, with a bandwidth of $D=$ $10^{-4} \Delta_{0}$, entailing a "bonding energy" of $\omega_{0} \simeq 0.008 \Delta_{0} \equiv$ $80 D$. They study two different interaction strengths: the first, $U=0.2 \Delta_{0}$, implies $J \simeq 12.7 D$ much larger than $D$ but much smaller than $U^{\mathrm{c}} \simeq 230 D$, thus matching the above definition of moderate coupling; the second, $U=4 \Delta_{0}$, implying $J \simeq 0.63 D$ slightly smaller than the bandwidth, is in the crossover region between moderate coupling and the Kondo regime. Fig. 5 shows LMA spec-

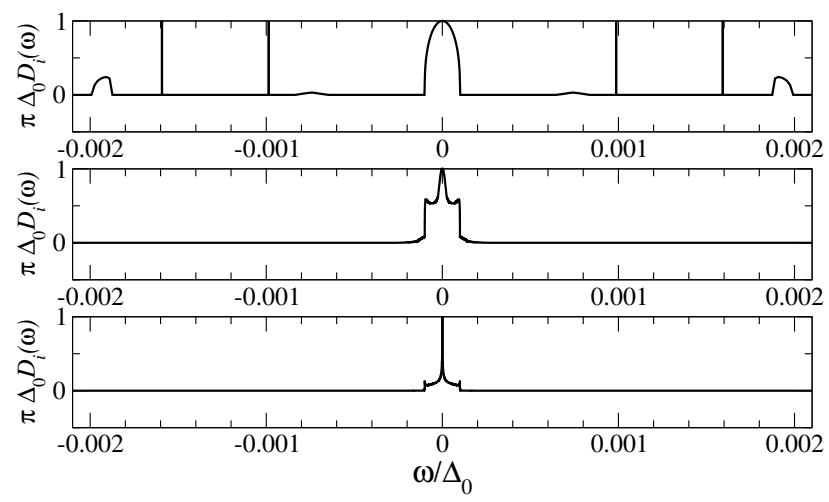

FIG. 5: Low-frequency sector of the LMA impurity spectra, $\left.\pi \Delta_{0} D_{(} \omega\right)$ vs. $\omega / \Delta_{0}$, for bandwidth $D=10^{-4} \Delta_{0}$, i.e., $\omega_{0} \simeq$ $0.008 \Delta_{0}=80 D$, and interaction strengths $U=0.2 \Delta_{0}$ (top), $U=4 \Delta_{0}$ (middle), and $U=10 \Delta_{0}$ (bottom). Discrete levels are represented by vertical lines. The Hubbard satellites at $|\omega| \simeq U / 2$ lie outside the plot range.

tra for the same parameters, along with an additional third calculation for $U=10 \Delta_{0}$ - which is in the true strong coupling regime difficult to reach for the Lanczos method.

Hofstetter and Kehrein's Lanczos spectrum for $U=$ $0.2 \Delta_{0}$ is dominated by Hubbard satellites at energies as high as $|\omega| \simeq \frac{U}{2}=0.1 \Delta_{0} \equiv 1000 D$ which are excluded from their plots. ${ }^{25}$ In the physically more relevant 
low-energy sector, their spectrum may be divided in two parts: (i) a central continuum for $|\omega| \leq D \equiv 10^{-4} \Delta_{0}$ which in shape is very similar to the Fermi-liquid band of the noninteracting limit; and (ii) two sets of narrow peaks on the antiferromagnetic scale $\mathcal{O}(J \simeq 12.7 D)$, the first located at $|\omega| \simeq 0.0018-0.0021 \Delta_{0}$ (i.e., $18-21 D$ ) and the second at $|\omega| \simeq 0.0025-0.0026 \Delta_{0}$ (i.e., $\left.25-26 D\right)$. Although it is not clear how these sets will evolve with a larger number of sites in the Lanczos calculations, a structure with at least two features will most likely prevail in this region.

The corresponding LMA results, displayed in the top panel of Fig. 5. reproduce - at least qualitatively - all characteristics of the Lanczos spectra: excellent agreement is found on the highest energy scale (off plot-range in the graph), governed by the Hubbard satellites at $\omega \simeq \pm 0.1 \Delta_{0}$ with almost $97 \%$ of the total spectral intensity; good agreement is also observed on the lowest energy scale $D$, occupied by the central Fermi-liquid continuum, of net weight $\mathcal{O}\left(D / \Delta_{0}\right) \sim 0.01 \%$, and essentially unrenormalized from the noninteracting-limit, since, throughout the whole continuum, the interaction self-energies $\tilde{\Sigma}_{\sigma}(\omega) \sim \mathcal{O}\left(U^{2} / \Delta_{0}\right)$ [eq. 39] ] are weak in comparison to the hybridization $\Delta(\omega) \sim \mathcal{O}\left(\Delta_{0}\right)$, and have thus very minor influence on the quasi-particle properties. Nevertheless, conceptually much simpler 2PT and UHF are similarly successful on the two latter energy scales.

On the intermediate energy scale, $J \simeq 12.7 D$, by contrast, where UHF and $2 \mathrm{PT}$ both predict a single set of levels (see Sec. IVB), solely the LMA produces a rich structure which qualitatively resembles the Lanczos spectra ${ }^{25}$ The dominant features are two sets of poles at $\omega= \pm \omega_{1,2}$ [eq. 41]]: the first, with $2 q_{1}=2.4 \%$ of the total spectral intensity and located at $|\omega|=\left|\omega_{1}\right| \simeq 1.28 \mathrm{~J} \simeq$ $0.0016 \Delta_{0} \equiv 16 D$, corresponds to shifted "orbital remnants"; the second, with $2 q_{2}=0.6 \%$ of the net spectral weight and situated at $|\omega|=\left|\omega_{2}\right| \simeq 0.78 J \simeq 0.001 \Delta_{0} \equiv$ $10 D$, is the novel feature related to resonant spin-flip processes (which has been discussed in the previous paragraph). Additionally, of the various sets of singleparticle bands arising from the self-energy continua (see Sec. III B, only the two most prominent are visible in the graph: the first, at $|\omega| \geq \omega_{\mathrm{m}} \simeq \frac{3}{2} J \simeq 0.0019 \Delta_{0} \equiv 19 D$ and of width $D$, stems from spin-flipping impurity scattering of the metallic host electrons close to the Fermi level - a process driving the Kondo physics in the strongcoupling limit $U \gg 4 \Delta_{0}$ (see Sec. IV D); a second, almost imperceptible set of continua of width $2 D$ is located at $|\omega| \geq \omega_{<} \simeq J / 2 \simeq 0.00064 \Delta_{0} \equiv 6.4 D$ and involves coupling the UHF orbitals to the low-lying spin-flip continua.

In summary, contrary to UHF and $2 \mathrm{PT}$, the LMA reproduces the main aspects of the Lanczos spectra also on the intermediate energy scale, but somewhat underestimates the antiferromagnetic exchange $J$. A better quantitative match would require a renormalized spin exchange constant $J^{\prime} \sim 1.6 J$, or equivalently a Coulomb coupling $U^{\prime}$ screened by the same factor for the spin chan- nel only.

For $U=4 \Delta_{0}$, not yet in the strong coupling regime, both the LMA and the Lanczos-determined spectra consist only of two appreciable contributions: the Hubbard satellites at $\omega \simeq \pm 2 \Delta_{0}$ on the high energy scale, and, on the other extreme, the low-energy Fermi-liquid continuum of width $\mathcal{O}(D)$ surrounding the Fermi level, $\omega=0$. The latter contribution, with its emergent central Kondo resonance, is plotted for the LMA in the middle panel of Fig. 5. The corresponding Lanczos graph by Hofstetter and Kehrein confirms this scenario, albeit with a slightly less developed Kondo resonance, and sharper peaks at the band edges $\omega= \pm D$. The overall agreement of the spectra is good, but could again be improved by renormalizing the LMA spin exchange, even though the mismatch might also partly stem from the artificial Lorentzian broadening or the small number of sites used in the Lanczos calculations.

Finally, the Fermi-liquid continuum for $U=10 \Delta_{0}$, plotted in the last panel of Fig. 5, illustrates the exponential narrowing of the Kondo resonance with interaction strength which is one of the hallmarks of the strong coupling regime to be discussed in the next section.

\section{Strong coupling: $U \gg 4 \Delta_{0}$}

In an AIM with a narrow metallic host, the singleparticle spectra suggest the following phenomenological definition of the strong coupling regime: an interaction strength belongs to the latter if (i) the metallic band at the Fermi level is dominated by a narrow central resonance whose shape is almost identical to Kondo resonances belonging to even larger interaction strengths; (ii) the Hubbard satellites appear as sharply peaked continua instead of discrete levels; and (iii) the spectrum contains no other visible features.

For $U=6 \Delta_{0}$ and $12 \Delta_{0}$, the LMA single-particle spectra do indeed comply with the last two of the above conditions. Whether however the shape of the Kondo resonance in the low-frequency sector of the spectra displayed in the bottom row of Fig. 6- is already scaling invariant is less obvious, but can be worked out in analogy to the AIM with an infinitely wide metallic host.19131 for $U \gg 4 \Delta_{0}$, and far from the band edges, $|\omega| \ll D$, the contributions to $G_{\sigma}(\omega)=\left[\omega-\Delta(\omega)-\tilde{\Sigma}_{\sigma}(\omega)\right]^{-1}$ of both, $\omega$ and $\Delta_{R}(\omega) \simeq 2 \Delta_{0} \omega / \pi D$, are negligible compared to the remaining two terms, given by $\Delta_{\mathrm{I}}(\omega) \simeq \Delta_{0}$, and the interaction self-energy $\tilde{\Sigma}_{\sigma}(\omega)$. In the frequency range considered here, the latter is proportional to $\Delta_{0}$ and scales in terms of the single variable $\tilde{\omega} \equiv \omega / \omega_{\mathrm{m}}$, with $\omega_{\mathrm{m}}(U) \simeq D \exp \left[-\pi U / 8 \Delta_{0}\right]$ [see eq. (40)]. This, in turn, leads to scaling invariance for the part of the singleparticle continuum closest to the Fermi level, $|\omega| \ll D$, i.e., for the central Kondo (or Abrikosov-Suhl) resonance.

The limiting curve for $U / \Delta_{0} \rightarrow \infty$, plotted with red dashes in Fig. 7] is identical for any symmetric AIM 

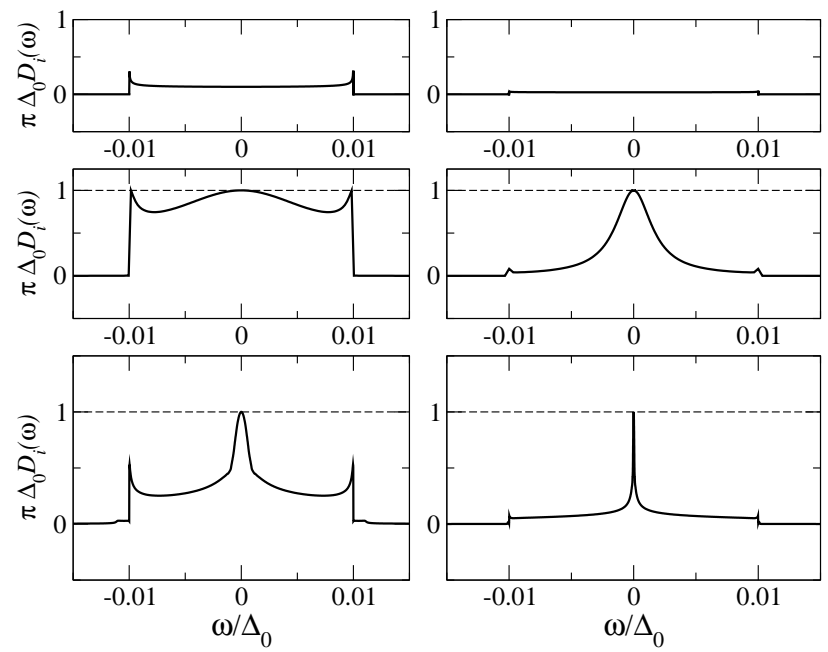

FIG. 6: Low-frequency impurity spectra, $\left.\pi \Delta_{0} D_{(} \omega\right)$ vs. $\omega / \Delta_{0}$, for UHF (top), 2PT (middle), and LMA (bottom), for bandwidth $D=0.01 \Delta_{0}$ and interaction strengths $U=6 \Delta_{0}$ (left column) and $U=12 \Delta_{0}$ (right column). The high-energy Hubbard satellites are far off plot range.

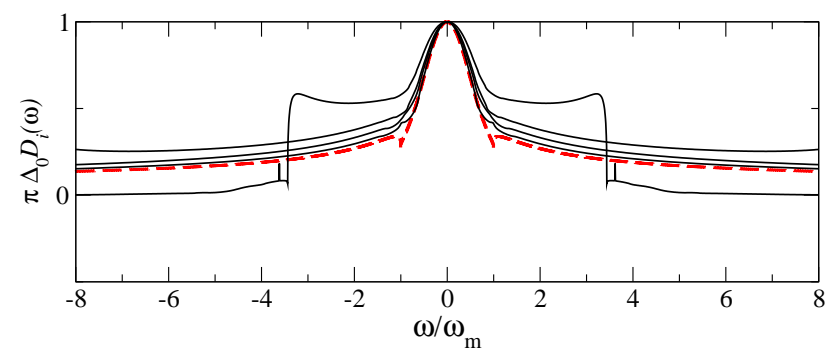

FIG. 7: Scaling of the Kondo resonance in the LMA impurity spectra, $\left.\pi \Delta_{0} D_{(} \omega\right)$ as a function of $\omega / \omega_{\mathrm{m}}$, with $\omega_{\mathrm{m}}$ the $(U-$ dependent) spin-flip or Kondo energy, for bandwidth $D=$ $0.01 \Delta_{0}$ and interaction strengths $U / \Delta_{0}=4,6,8,12$ (solid lines from top to bottom), and in the limit $U / \Delta_{0} \rightarrow \infty$ (red dashed line). Full discussion in text.

with a metallic host. It comprises two parts, the first being the narrow central region between the cusps at $|\omega| \simeq \omega_{\mathrm{m}}$, within which the line shape mostly carries the Lorentzian signature of Landau quasi-particles of weight $Z=\left\{1-[\partial \operatorname{Re} \Sigma(\omega) / \partial \omega]_{\omega=0}\right\}^{-1}$, while the second region, outside the cusps, is characterized by long logarithmically decaying spectral tails ${ }^{1952}$ (The cusps in the spectrum at $|\omega| \simeq \omega_{\mathrm{m}}$ are artefacts stemming from the RPA-like structure of the polarization propagator and can be removed by a more realistic ansatz for the latter, ${ }^{31}$ producing LMA line shapes which then excellently agree with corresponding NRG data ${ }^{19}$ )

The graphs in the first two rows of Fig. 6 show clearly that such subtle effects are out of reach for the other two methods studied here: UHF, on the one hand, suppresses for $U / \Delta_{0} \rightarrow \infty$ all spectral intensity close to the
Fermi level as a result of an incipient transition towards an insulating atomic-limit state; 2PT, on the other hand, correctly captures the persistent metallic character of the system - manifest in the Friedel sum rule pinning of the spectra at the Fermi level - but misses the exponential narrowing and the non-Lorentzian shape of the Kondo resonance.

Relatedly, on the high energy scale $|\omega| \sim U$, the LMA correctly predicts sharply peaked Hubbard bands instead of genuine levels, ensuing from the absorption of the Hubbard "levels" at $|\omega| \simeq \frac{U}{2}+\frac{3 J}{2}$ by their former "side bands" at $\frac{U}{2} \lesssim|\omega| \lesssim \frac{U}{2}+2 D$; again, UHF and $2 \mathrm{PT}$ both fail to catch this many-body broadening effect.

Finally, all methods - UHF, 2PT, LMA, and also Lanczos - agree in the large spectral gap ranging from the low-energy continuum of width $D$ to the high-energy Hubbard satellites. Especially the orbital remnants, which in weak and moderate coupling are found inside this gap, are now missing. A more rigorous analysis shows that they in fact still exist, occurring exponentially close to the band edges $|\omega|=D$, but are undetectable because their spectral weight vanishes exponentially.

\section{CONCLUSIONS}

In this article, a symmetric AIM with a narrow metallic host has been studied with three different theoretical approaches: UHF, 2PT, and the LMA.

The persistent metallic character of the system which renders diagrammatic perturbation expansions (like $2 \mathrm{PT}$ ) viable in the first place - entails that its dynamics hinges on the impurity Coulomb repulsion $U$ and the noninteracting Green function $g(\omega)$. The latter, in turn, depends solely on the hybridization $\Delta(\omega)$, which, for the present case of a narrow host band, is mainly determined by its width $D$ and strength at the Fermi level $\Delta_{0}$. Despite this reduced set of ultimately three parameters $-\Delta_{0}, D$, and $U$ - the single-particle spectra are found to be rich, in particular in the physically relevant low-energy sector close to the Fermi surface, indicating the competition of various physical processes and their associated energy scales. These encompass Fermi-liquid behaviour within the low-energy continuum of width $D$, molecular orbital formation related to the bonding energy $\omega_{0} \sim \sqrt{\Delta_{0} D}$, antiferromagnetic phenomena driven by the exchange coupling $J=4 \omega_{0}^{2} / U$, and finally the Kondo effect with its magnetic scale $\omega_{\mathrm{m}} \simeq D \exp \left[-\pi U / 8 \Delta_{0}\right]$.

Up to moderate interaction strengths, $U \lesssim \Delta_{0}$, the low-energy physics is dominated by the integrated hopping between host and impurity, $\omega_{0}^{2}=\frac{1}{\pi} \int \mathrm{d} \omega \Delta_{\mathrm{I}}(\omega)$, and the antiferromagnetic exchange, $J$, which are both insensitive to the details of the hybridization function. The precise form of $\Delta(\omega)$ therefore only determines the essentially unperturbed metallic band surrounding the Fermi level. In the opposite limit of large interactions, $U \gg \Delta_{0}$, the low-energy physics is governed by the Kondo effect. This leads to a spectral scaling, in terms of $\omega / \omega_{\mathrm{m}}$, which, 
again, does not depend on any details of the hybridization function.

The LMA has been shown to produce meaningful results over the entire range of interaction strengths. For small interactions, $U \lesssim \omega_{0}$, it predicts a vanishing impurity moment and connects smoothly to 2PT, yielding single-particle spectra carrying the signature of prevailing orbital physics.

For moderate coupling strengths, $\omega_{0} \ll U \lesssim \Delta_{0}$ (or alternatively $\left.D \ll J \ll \omega_{0}\right)$, in addition to the highenergy Hubbard levels and the essentially unrenormalized Fermi-liquid continuum on the lowest energy scale, the LMA produces rich spectra on the $J$-scale: here, two pairs of poles along with several accompanying sidebands can be observed. Similar structures were found in corresponding Lanczos-determined spectra by Hofstetter and Kehrein. 25 Their main contributions can be rationalized as follows: one pair of poles may be considered as remnants of the molecular bonding and anti-bonding orbitals which similarly occur in UHF and 2PT; the other pair - which misses in the latter approaches and also in other state-of-the art techniques like, e.g., slave-boson based methods 47 - is a somewhat unexpected feature and arises due to resonant collective spin-flip processes between the impurity and the UHF orbital remnants. Similar spectral contributions, in the form of sharp resonances at the inner band edges of the Hubbard satellites, have been observed numerically in the metallic phase close to the Mott transition, which occurs in the infinitedimensional Hubbard model $!^{27}[29$ These features have been dubbed antipolarons by Karski et al. who suspect them, mainly on energetic grounds, to take their origin in bonding/antibonding phenomena between heavy quasiparticles and collective spin excitations. $\stackrel{28}{\text { Although }}$ the same authors admit in a subsequent publication 29 that this "complex composite excitation is not yet understood," their original idea is supported by the above interpretation of the corresponding AIM features. Karski et al.'s results for the Hubbard model clearly indicate that sharp features will prevail in the presence of host or bath correlations, although their aspect may naturally be altered, especially if they coincide with another band.

In the limit of large interaction strengths, $U \gg \Delta_{0}$, of the three investigated methods only the LMA captures simultaneously the many-body broadening of the high-energy Hubbard satellites and the relevant lowenergy phenomena embodied in the exponentially narrowing Abrikosov-Suhl or Kondo resonance with its distinct logarithmically decaying wings $\frac{19 \mid 32}{}$ Moreover, the observed Kondo resonance has been shown to possess the universal shape and scaling properties characteristic for symmetric AIMs with a metallic host.

The fairly accurate analytic solutions obtained within the framework of the LMA suggest a classification into two regimes, which cover almost the entire range of interactions: the first englobes weak and moderate interactions, $U \lesssim \Delta_{0}$, and its Fermi-liquid properties are primarily inherited from the noninteracting system, while its orbital contributions follow directly from the two-site approximation where the narrow host is treated as a single site; in marked contrast to the latter is the second regime, suitable for large interactions, $U \gg \Delta_{0}$, where the narrow host band behaves as infinitely wide in comparison to the exponentially small Kondo energy.

\section{Acknowledgments}

First and foremost, I would like to thank David Logan for drawing my attention to the subject and for countless valuable discussions. I am also most grateful to Florian Gebhard, Matthew Glossop, Nigel Dickens, Adam Kirrander, Roland Hayn, and Laurent Raymond for constructive comments and remarks. The present work was initiated at the Physical and Theoretical Chemistry Laboratory, Oxford, whom I would like to thank for their hospitality, and with financial support of the Deutscher Akademischer Austauschdienst (DAAD) under Grant No. D/98/27069.
1 P. W. Anderson, Phys. Rev. 124, 41 (1961).

2 S. Sasaki, S. De Franceschi, J. M. Elzerman, W. G. van der Wiel, M. Eto, S. Tarucha, and L. P. Kouwenhoven, Nature (London) 405, 764 (2000).

3 W. G. van der Wiel, S. De Franceschi, T. Fujisawa, J. M. Elzerman, S. Tarucha, and L. P. Kouwenhoven, Science 289, 2105 (2000).

4 T. W. Odom, J.-L. Huang, C. L. Cheung, and C. M. Lieber, Science 290, 1549 (2000).

5 J. Nygård, D. H. Cobden, and P. E. Lindelof, Nature (London) 408, 342 (2000).

${ }^{6}$ A. Zhao, Q. Li, L. Chen, H. Xiang, W. Wang, S. Pan, B. Wang, X. Xiao, J. Yang, J. G. Hou., et al., Science 309, 1542 (2005).

7 D. Goldhaber-Gordon, H. Shtrikman, D. Mahalu, D. Abusch-Magder, U. Meirav, and M. A. Kastner, Na- ture (London) 391, 156 (1998).

8 W. Liang, M. P. Shores, M. Bockrath, J. R. Long, and H. Park, Nature (London) 417, 725 (2002).

9 A. C. Hewson, The Kondo Problem to Heavy Fermions (Cambridge University Press, Cambridge, England, 1993).

10 A. M. Tsvelik and P. B. Wiegmann, Advances in Physics 32, 453 (1983).

11 A. Okiji and N. Kawakami, Phys. Rev. Lett. 50, 1157 (1983).

12 P. Schlottmann, Physics Reports 181, 1 (1989), ISSN 0370-1573.

${ }^{13}$ H. R. Krishna-murthy, J. W. Wilkins, and K. G. Wilson, Phys. Rev. B 21, 1003 (1980).

14 H. R. Krishna-murthy, J. W. Wilkins, and K. G. Wilson, Phys. Rev. B 21, 1044 (1980).

${ }^{15}$ H. O. Frota and L. N. Oliveira, Phys. Rev. B 33, 7871 
(1986).

16 O. Sakai, Y. Shimizu, and T. Kasuya, J. Phys. Soc. Japan 58, 3666 (1989).

17 T. A. Costi and A. C. Hewson, Physica B 163, 179 (1990).

18 W. Hofstetter, Phys. Rev. Lett. 85, 1508 (2000).

19 N. L. Dickens and D. E. Logan, J. Phys.: Condens. Matter 13, 4505 (2001).

20 M. R. Galpin and D. E. Logan, J. Phys.: Condens. Matter 17, 6959 (2005).

21 R. Bulla, T. A. Costi, and T. Pruschke, Rev. Mod. Phys. 80, 395 (2008).

${ }^{22}$ W. Metzner and D. Vollhardt, Phys. Rev. Lett. 62, 324 (1989).

23 A. Georges, G. Kotliar, W. Krauth, and M. J. Rozenberg, Rev. Mod. Phys. 68, 13 (1996).

${ }^{24}$ F. Gebhard, The Mott Metal-Insulator Transition, vol. 137 of Springer Tracts in Modern Physics (Springer, Berlin, 1997).

${ }^{25}$ W. Hofstetter and S. Kehrein, Phys. Rev. B 59, R12732 (1999).

26 S. Kehrein, Phys. Rev. Lett. 81, 3912 (1998).

27 S. Nishimoto, F. Gebhard, and E. Jeckelmann, J. Phys.: Condens. Matter 16, 7063 (2004).

28 M. Karski, C. Raas, and G. S. Uhrig, Phys. Rev. B 72, 113110 (2005).

29 M. Karski, C. Raas, and G. S. Uhrig, Phys. Rev. B 77, 075116 (2008).

30 S. Schäfer and D. E. Logan, Phys. Rev. B 63, 045122 (2001).

31 D. E. Logan, M. P. Eastwood, and M. A. Tusch, J. Phys.: Condens. Matter 10, 2673 (1998).

32 M. T. Glossop and D. E. Logan, J. Phys.: Condens. Matter 14, 6737 (2002).

33 M. R. Galpin and D. E. Logan, Eur. Phys. J. B 62, 129 (2008)

34 D. E. Logan and M. T. Glossop, J. Phys.: Condens. Matter 12, 985 (2000).

35 D. E. Logan and N. L. Dickens, J. Phys.: Condens. Matter 13, 9713 (2001).
36 D. E. Logan and N. L. Dickens, Europhys. Lett. 54, 227 (2001).

37 V. E. Smith, D. E. Logan, and H. R. Krishnamurthy, Eur. Phys. J. B 32, 49 (2003).

38 D. E. Logan and N. S. Vidhyadhiraja, J. Phys.: Condens. Matter 17, 2935 (2005).

39 M. R. Galpin, A. B. Gilbert, and D. E. Logan, J. Phys.: Condens. Matter 21, 375602 (2009).

40 E. Lange, Mod. Phys. Lett. B 12, 915 (1998).

41 D. C. Langreth, Phys. Rev. 150, 516 (1966).

42 K. Takegahara, Y. Shimizu, and O. Sakai, J. Phys. Soc. Japan 61, 3443 (1992).

43 K. Chen and C. Jayaprakash, Phys. Rev. B 57, 5225 (1998).

44 M. R. Galpin and D. E. Logan, Phys. Rev. B 77, 195108 (2008).

45 J. M. Luttinger, Phys. Rev. 121, 942 (1961).

46 P. W. Anderson, J. Phys. C: Solid State Phys. 3, 2436 (1970).

47 L. Vaugier, A. A. Aligia, and A. M. Lobos, Phys. Rev. B 76, 165112 (2007).

48 S. Doniach and M. Sunjić, J. Phys. C: Solid State Phys. 3, 2850 (1970).

49 A. C. Seridonio, L. N. Oliveira, and M. Yoshida, Braz. J. Phys. 39, 226 (2009).

50 A. Rosch, T. A. Costi, J. Paaske, and P. Wölfle, Phys. Rev. B 68, 014430 (2003).

51 In the present particle-hole symmetric AIM, where the impurity level is chained to the interaction strength via $\varepsilon_{i}=-U / 2$, it is convenient to include $\varepsilon_{i}$ in the self-energies $\tilde{\Sigma}_{\sigma}(\omega)$ instead of the noninteracting $g(\omega)$. For the general (asymmetric) AIM, where $\varepsilon_{i}$ and $U$ are truly independent, the opposite choice, used in Ref. 32 , is appropriate.

52 These spectral tails have formerly been considered to be of Doniach-S̆unjić form,$\frac{15] 48 / 49}{2}$ governed by an asymptotic algebraic falloff $\left(|\omega|^{-1 / 2}\right)$, instead of the actual logarithmic behaviour! $19 \mid 32 / 50$ 